\title{
Discovery prospects of a light scalar in the NMSSM
}

\author{
Ulrich Ellwanger ${ }^{a, b}$ and Matías Rodríguez-Vázquez ${ }^{a}$ \\ ${ }^{a}$ Laboratoire de Physique Théorique, UMR 8627, CNRS, \\ Université de Paris-Sud, Université Paris-Saclay, \\ Bât. 210, 91405 Orsay, France \\ ${ }^{b}$ School of Physics and Astronomy, University of Southampton, \\ Highfield, Southampton SO17 1BJ, U.K. \\ E-mail: ulrich.ellwanger@th.u-psud.fr, \\ matias.rodriguez-vazquez@th.u-psud.fr
}

ABSTRACT: We study a region in the NMSSM parameter space in which the mass of the SM-like Higgs boson is uplifted by $\sim 4-17 \mathrm{GeV}$, allowing for stop masses and $\left|A_{t}\right| \leq 1 \mathrm{TeV}$ alleviating the little fine tuning problem of the MSSM. An uplift of the mass of the SM-like Higgs boson is possible in two distinct regions in the NMSSM parameter space: either for large $\lambda$ and small $\tan \beta$ or, through singlet-doublet mixing, for small $\lambda$ and large $\tan \beta$. For a mostly singlet-like Higgs state $H_{S}$ with a mass below $125 \mathrm{GeV}$ we investigate possible direct or indirect search channels at the run II of the LHC as function of the NMSSM-specific uplift of the mass of the SM-like Higgs boson: direct production of $H_{S}$ in gluon fusion with $H_{S}$ decaying into diphotons, modified reduced couplings of the SM-like Higgs state, and the possible production of $H_{S}$ in $g g F \rightarrow A \rightarrow Z+H_{S}$. We find that the region featuring singletdoublet mixing can be tested if searches at the LHC at $13 \mathrm{TeV}$ for BSM Higgs bosons in the mass range $88-102 \mathrm{GeV}$ decaying into diphotons become sensitive to signal cross sections $\sigma\left(g g \rightarrow H_{S} \rightarrow \gamma \gamma\right) \sim 20 \mathrm{fb}$, or if measurements of the reduced coupling $\kappa_{V}\left(H_{S M}\right)$ of the SM Higgs boson to electroweak gauge boson exclude (or confirm) the region $\kappa_{V}\left(H_{S M}\right) \lesssim 0.93$.

Keywords: Supersymmetry Phenomenology

ArXiv EPrint: 1512.04281 


\section{Contents}

1 Introduction 1

2 The neutral Higgs sector of the NMSSM 4

3 Numerical analysis $\quad 8$

4 Results 9

4.1 Searches for $H_{S}$ in the diphoton final state 9

$\begin{array}{lll}4.2 & \text { Reduced couplings of } H_{S M} & 12\end{array}$

$\begin{array}{lll}4.3 & H_{S} \text { production via decays of heavy states } H / A & 14\end{array}$

$\begin{array}{llr}5 & \text { Conclusions } & 17\end{array}$

\section{Introduction}

Since the discovery in 2012 of a SM-like Higgs scalar with a mass close to $125 \mathrm{GeV}$ by the ATLAS [1] and CMS [2] collaborations, its couplings to gauge bosons and fermions have been measured with an unexpectedly high precision, see [3] for a recent combination of the measurements of ATLAS and CMS. These confirm essentially the couplings expected from the Standard Model (SM).

Within supersymmetric extensions of the SM one finds an enlarged Higgs sector featuring additional neutral CP-even, CP-odd and charged states. It is relatively natural within the parameter space of supersymmetric extensions of the SM to find a neutral CP-even Higgs state with couplings to gauge bosons and fermions very close to the ones expected from the SM. However, within the Minimal Supersymmetric extension of the SM (MSSM) the mass of $\sim 125 \mathrm{GeV}$ of this SM-like Higgs state is not easy to explain. At tree level the mass of the SM-like Higgs state is bounded from above by $M_{Z}$, and accordingly large radiative corrections requiring large scalar top (stop) masses and/or mass splittings well above $1 \mathrm{TeV}$ are needed in order to uplift the mass of the SM-like Higgs state from $M_{Z}$ to $\sim 125 \mathrm{GeV}[4-11]$.

But heavy stop masses/mass splittings lead to large radiative corrections to a soft Susy breaking Higgs mass term, which has to be tuned against the $\mu$ parameter if much larger than $M_{Z}$ (see [12] and refs. therein). Accordingly the Higgs mass of about $125 \mathrm{GeV}$ aggravates a little finetuning problem within the MSSM, pointed out already in the context of LEP bounds on the Higgs mass in [13-16].

It is known that the upper tree level bound of $M_{Z}$ on the mass of the SM-like Higgs boson does not hold in the Next-to-Minimal Supersymmetric extension of the SM (NMSSM, see $[17,18]$ for reviews). Accordingly the NMSSM can alleviate the little finetuning problem of the MSSM [11, 12, 19-28]. It shares the benefits of supersymmetric extensions 
of the Standard Model (SM) with the MSSM: the hierarchy problem can be strongly reduced, the presence of dark matter can be explained, and the running gauge couplings are automatically consistent with a Grand Unified Theory (GUT).

In the NMSSM, an additional gauge singlet superfield $\widehat{S}$ couples with a dimensionless coupling $\lambda$ to the two SU(2) doublet superfields $\widehat{H}_{u}$ and $\widehat{H}_{d}$ of the MSSM. A vacuum expectation value of the scalar component of $\widehat{S}$ generates dynamically a $\mu$ parameter of the order of the Susy breaking scale, solving the $\mu$-problem of the MSSM [29]. The NMSSM spectrum contains three neutral CP-even Higgs scalars. Typically, one of them is mostly SM-like (denoted by $H_{S M}$ in the following), one has the properties of the (heavy) MSSM-like state $H$, and a third state $H_{S}$ is mostly singlet-like. These states are mixtures of the weak eigenstates (the scalar components of $\widehat{H}_{u}, \widehat{H}_{u}$ and $\widehat{S}$ ). Past and present searches for Higgs bosons at LEP, the Tevatron and the LHC do not exclude masses of $H_{S}$ below $125 \mathrm{GeV}$.

In fact, in the NMSSM two distinct mechanisms can lead to additional tree level contributions to the mass of the SM-like state $H_{S M}$ :

a) If $\lambda$ is large enough $\left(\lambda^{2}>\left(g_{1}^{2}+g_{2}^{2}\right) / 2\right.$, where $g_{1}$ and $g_{2}$ are the electroweak gauge couplings) and $\tan \beta$ is small enough $\left(\tan \beta \lesssim 6\right.$ ), the additional quartic coupling $\sim \lambda^{2} H_{S M}^{4}$ in the scalar potential lifts its mass above $M_{Z}$. However, $\lambda \gtrsim 1$ (so-called $\lambda$ Susy [30, 31]) would be required in order to push the tree level mass from $M_{Z}$ to $125 \mathrm{GeV}$ in which case $\lambda$ runs into a Landau singularity well below the GUT scale. In order to avoid this we confine ourselves subsequently to $\lambda \lesssim 0.75$.

b) If the mostly singlet-like state $H_{S}$ has a mass below $125 \mathrm{GeV}$, mixing between $H_{S}$ and $H_{S M}$ (more precisely, among the weak eigenstates) leads to an increase of the mass of the latter. The impact of such mixings on the Higgs spectrum of the NMSSM has been known for a while [23, 24, 32-35], but became particularly interesting once the mass of $\sim 125 \mathrm{GeV}$ of the mostly SM-like state had been measured [4, 26, 36-58]. The mass shift of up to $\sim 8 \mathrm{GeV}$ occurs now mostly for large $\tan \beta$ and smaller $\lambda \approx 0.04-0.1$, the latter in order to avoid constraints from LEP on a Higgs-like state with a mass below $\sim 114 \mathrm{GeV}$ [59]. (The increase of the mass of the SM-like state $H_{S M}$ through mixing implies a decrease of the lighter singlet-like state $H_{S}$.) Hence the corresponding region in parameter space is clearly distinct from the one where the quartic SM-Higgs self coupling is enhanced.

In the present paper we consider both possibilities, but confine ourselves to the case where the mass of the mostly singlet-like state $H_{S}$ is below $125 \mathrm{GeV}$ : this situation is preferred also in the large $\lambda$-small $\tan \beta$ regime, since singlet-doublet mixing would always imply a decrease of the mass of the SM-like state if the singlet-like state is heavier, and mixing is hard to avoid if $\lambda$ is large (unless $H_{S}$ is very heavy and/or the corresponding off-diagonal element in the mass matrix happens to be small). On the other hand a mass of the mostly singlet-like state $H_{S}$ below $\sim 60 \mathrm{GeV}$ would lead to dominant decays of $H_{S M}$ into pairs of $H_{S}$ unless $\lambda$ (and hence the mixing angle) is very small; also the LEP constraints are quite strong for this mass range [59]. We found that a sizeable positive mass shift for the SM-like state is unlikely here.

It is known that singlet-doublet mixing has two distinct phenomenological consequences: 
a) The mostly singlet-like state inherits couplings to SM gauge bosons and fermions from the SM-like state proportional to the (sinus of the) mixing angle. This leads to nonvanishing production cross sections for $H_{S}$, and its potential discovery at the LHC.

b) Simultaneously, the couplings of $H_{S M}$ to gauge bosons and fermions get reduced. The uncertainties of the measured couplings of $H_{S M}$ at the run I of the LHC [3] are expected to decrease further after measurements at the run II $[60,61]$.

It is the purpose of the present paper to study in how far the combination of both sources of future information can constrain the presence - or lead to a discovery - of a light singlet-like Higgs boson in the NMSSM, as function of the NMSSM specific mass shift of the SM-like state. We also indicate the possible production of $H_{S}$ in decays of heavier MSSM-like $H / A$ states. To start with, we have to collect the available constraints on this scenario from LEP and measurements at the run I of the LHC.

First, bounds on couplings to the $Z$ boson times the branching fraction of an additional light Higgs boson into $b \bar{b}$ and gluons originate from LEP [59].

Second, limits originate from direct searches for extra (lighter) Higgs states in the diphoton channel by ATLAS [62] and CMS [63]: despite the relatively small diphoton branching fraction this final state is the most promising one to search for, in particular in view of the possibility that the diphoton branching fraction of $H_{S}$ can be considerably larger than the one of a SM-like Higgs boson of corresponding mass [43, 45, 54, 57, 58, 64-69].

Third, limits originate from the potential reduction of couplings of $H_{S M}$ to SM gauge bosons and fermions through mixing with a gauge singlet. The corresponding measurements of production and decay mode dependent signal strengths of ATLAS and CMS have recently been combined by the collaborations in [3]. Global fits to the couplings (or the coupling modifiers) require, in principle, likelihood grids including information on deviations from Gaussianity and correlations among uncertainties in particular for identical final states from different production modes. Moreover such global fits depend crucially on the assumptions on the underlying model like custodial symmetry (identical modifications of couplings to $W$ and $Z$ bosons), correlated modifications of couplings to $b$ quarks and $\tau$ leptons like in specific Higgs doublet models, and possible additional contributions to loop induced couplings to gluons and photons.

The latest global fits including assumptions corresponding to the NMSSM (custodial symmetry, correlated modifications of couplings to $b$ quarks and $\tau$ leptons, possible additional contributions notably to the loop induced coupling to photons) have been performed in [70]. We have checked that their combined signal strengths are very close to the ones in [3] and use, for the scan of the NMSSM parameter space (see below), their 95\% CL on signal strengths of $H_{S M}$ (verifying only subsequently the bounds from [3]). Electroweak precision data (the $W$ boson mass) do not constrain the parameter space of the NMSSM with a light $H_{S}$ [71]. Overall, in the NMSSM the experimental constraints on the $H_{S M}-H_{S}$ mixing angle (for $M_{H_{S}}$ below $125 \mathrm{GeV}$ ) are similar to the ones obtained from studies within simple singlet-extensions of the non-supersymmetric SM [56, 72-75].

As a next step we study in how far future measurements of diphoton signal rates (via ggF) of $H_{S}$ at $13 \mathrm{TeV}$ are sensitive to the NMSSM specific mass shift of the SM-like state. Likewise, the dependence of the couplings of $H_{S M}$ (and hence of the $H_{S M}-H_{S}$ mixing angle) on the NMSSM specific mass shift of the SM-like state is analysed. The 
results clarify in how far the NMSSM specific mass shift can be tested in the future, and which of the different measurements are potentially more sensitive. Studies of possible $H_{S}$ diphoton signal rates (after the discovery of the $H_{S M}$ state) in the NMSSM have been performed earlier in [43, 45, 57, 58,69] (see also [56]), and correlations with mass shifts (from $H_{S M}-H_{S}$ mixing only) have been presented in [52]. In the present paper we extend the studies of such correlations including the large $\lambda$-small $\tan \beta$ regime, include constraints from ATLAS [62] and CMS [63] from direct searches for lighter Higgs states in the diphoton channel, and obtain possible $H_{S}$ diphoton signal rates which partially deviate from (are larger than) the ones obtained earlier.

In the next section we recall the properties of the Higgs sector of the NMSSM relevant for the present study, and define a NMSSM specific mass shift $\Delta_{\text {NMSSM }}$ of the SM-like Higgs state. In section 3 we describe the scans over the parameter space. In section 4 we present the results of the scans as function of $\Delta_{\mathrm{NMSSM}}: H_{S}$ diphoton signal rates at 8 and $13 \mathrm{TeV}$ c.m. energy, modifications of the couplings of $H_{S M}$, and correlations among them. We discuss and compare prospects for tests of the scenarios under study, including the possible production of $H_{S}$ in decays of heavy MSSM-like $H / A$ states. Finally we conclude in section 5 .

\section{The neutral Higgs sector of the NMSSM}

In this paper we consider the CP-conserving $\mathbb{Z}_{3}$-invariant NMSSM. The superpotential of the NMSSM Higgs sector reads

$$
W_{\text {Higgs }}=\lambda \hat{S} \hat{H}_{u} \cdot \hat{H}_{d}+\frac{\kappa^{3}}{3} \hat{S}^{3}
$$

where $\hat{S}$ is the chiral singlet superfield. Once the real component of the superfield $\hat{S}$ develops a vacuum expectation value (vev) $s$, the first term in the superpotential generates an effective $\mu$ term

$$
\mu=\lambda s .
$$

The soft Higgs-dependent SUSY breaking terms are

$$
\mathcal{L}_{\text {Soft }}=-m_{H_{u}}^{2}\left|H_{u}\right|^{2}-m_{H_{d}}^{2}\left|H_{d}\right|^{2}-m_{S}^{2}|S|^{2}-\left(\lambda A_{\lambda} H_{u} \cdot H_{d} S+\frac{1}{3} \kappa A_{\kappa} S^{3}+\text { h.c }\right) .
$$

Then, from the SUSY gauge interactions, the $F$ and soft SUSY breaking terms one obtains the Higgs potential

$$
\begin{aligned}
V= & \left|\lambda\left(H_{u}^{+} H_{d}^{-}-H_{u}^{0} H_{d}^{0}\right)+\kappa S^{2}\right|^{2} \\
& +\left(m_{H_{u}}^{2}+|\mu+\lambda S|^{2}\right)\left(\left|H_{u}^{0}\right|^{2}+\left|H_{u}^{+}\right|^{2}\right)^{2}+\left(m_{H_{d}}^{2}+|\mu+\lambda S|^{2}\right)\left(\left|H_{d}^{0}\right|^{2}+\left|H_{d}^{-}\right|^{2}\right)^{2} \\
& \frac{g_{1}^{2}+g_{2}^{2}}{8}\left(\left|H_{u}^{0}\right|^{2}+\left|H_{u}^{+}\right|^{2}-\left|H_{d}^{0}\right|^{2}-\left|H_{d}^{-}\right|^{2}\right)^{2}+\frac{g_{2}^{2}}{2}\left|H_{u}^{+} H_{d}^{0 *}+H_{u}^{0} H_{d}^{-*}\right|^{2} \\
& +m_{S}^{2}|S|^{2}+\left(\lambda A_{\lambda}\left(H_{u}^{+} H_{d}^{-}-H_{u}^{0} H_{d}^{0}\right) S+\frac{1}{3} \kappa A_{\kappa} S^{3}+\text { h.c. }\right) .
\end{aligned}
$$


After expanding around the vacuum expectation values $v_{u}, v_{d}$ and $s$ (which can be taken to be real and positive), the Higgs fields are given by

$$
\begin{aligned}
H_{u} & =\left(\begin{array}{c}
H_{u}^{+} \\
H_{u}^{0}=v_{u}+\frac{1}{\sqrt{2}}\left(H_{u, r}^{0}+i H_{u, i}^{0}\right)
\end{array}\right), \quad H_{d}=\left(\begin{array}{c}
H_{d}^{0}=v_{d}+\frac{1}{\sqrt{2}}\left(H_{d, r}^{0}+i H_{d, i}^{0}\right) \\
H_{d}^{-}
\end{array}\right), \\
S & =s+\frac{1}{\sqrt{2}}\left(S_{r}+i S_{i}\right) .
\end{aligned}
$$

Once the soft Higgs masses are expressed in terms of $M_{Z}, \tan \beta$ and $s$ using the minimization equations of the potential, the Higgs sector of the NMSSM at tree level is described by six parameters

$$
\lambda, \quad \kappa, \quad \tan \beta, \quad \mu=\lambda s, \quad A_{\lambda} \text { and } A_{\kappa} .
$$

Defining $v^{2}=2 M_{Z}^{2} /\left(g_{1}^{2}+g_{2}^{2}\right) \sim(174 \mathrm{GeV})^{2}$, the $3 \times 3$ CP-even mass matrix in the basis $\left(H_{d, r}, H_{u, r}, S_{r}\right)$ reads:

$$
\begin{aligned}
& \mathcal{M}_{S, 11}^{2}=M_{Z}^{2} \cos ^{2} \beta+\mu\left(A_{\lambda}+\kappa s\right) \tan \beta, \\
& \mathcal{M}_{S, 12}^{2}=\left(\lambda v^{2}-\frac{M_{Z}^{2}}{2}\right) \sin 2 \beta-\mu\left(A_{\lambda}+\kappa s\right), \\
& \left.\mathcal{M}_{S, 13}^{2}=\lambda v\left(2 \mu \cos \beta-\left(A_{\lambda}+2 \kappa s\right) \sin \beta\right)\right), \\
& \mathcal{M}_{S, 22}^{2}=M_{Z}^{2} \sin ^{2} \beta+\mu\left(A_{\lambda}+\kappa s\right) \cot \beta+\Delta_{\text {rad }}, \\
& \left.\mathcal{M}_{S, 23}^{2}=\lambda v\left(2 \mu \sin \beta-\left(A_{\lambda}+2 \kappa s\right) \cos \beta\right)\right), \\
& \mathcal{M}_{S, 33}^{2}=\lambda A_{\lambda} \frac{v^{2}}{2 s} \sin 2 \beta+\kappa s\left(A_{\kappa}+4 \kappa s\right) .
\end{aligned}
$$

Here $\Delta_{\text {rad }}$ denotes the dominant radiative corrections due to top/stop loops,

$$
\Delta_{\mathrm{rad}}=\frac{3 m_{t}^{4}}{4 \pi^{2} v^{2}}\left(\ln \left(\frac{m_{T}^{2}}{m_{t}^{2}}\right)+\frac{X_{t}^{2}}{m_{T}^{2}}\left(1-\frac{X_{t}^{2}}{12 m_{T}^{2}}\right)\right)
$$

where $m_{T}$ is the geometrical average of the soft SUSY breaking stop masses, and $X_{t}=$ $A_{t}-\mu / \tan \beta$ with $A_{t}$ the soft SUSY breaking stop trilinear coupling.

It is convenient to rotate $\mathcal{M}_{S}^{2}$ by an angle $\beta$ in the doublet sector sector into $\mathcal{M}_{S}^{\prime 2}$ in the basis $H_{S M}^{\prime}, H^{\prime}, S^{\prime}$ (with $S^{\prime} \equiv S_{r}$ ):

$$
\mathcal{M}_{S}^{\prime 2}=R(\beta) \mathcal{M}_{S}^{2} R^{\mathcal{T}}(\beta), \quad R(\beta)=\left(\begin{array}{ccc}
\cos \beta & \sin \beta & 0 \\
\sin \beta & -\cos \beta & 0 \\
0 & 0 & 1
\end{array}\right) .
$$

Such a basis (also known as Higgs basis) has the advantage that only the component $H_{S M}^{\prime}$ of the Higgs doublets acquires a vev $v$ and that, for realistic parameters, it is nearly diagonal: $H_{S M}^{\prime}$ has SM-like couplings to fermions and electroweak gauge bosons, the heavy doublet field $H^{\prime}$ is the CP-even partner of the MSSM-like CP-odd state $A$, while $S^{\prime}$ remains a pure singlet. The mass matrix $\mathcal{M}_{S}^{\prime 2}$ in the basis $\left(H_{S M}^{\prime}, H^{\prime}, S^{\prime}\right)$ has the elements

$$
\mathcal{M}_{S, 11}^{\prime 2}=M_{Z}^{2} \cos ^{2} 2 \beta+\lambda^{2} v^{2} \sin ^{2} 2 \beta+\sin ^{2} \beta \Delta_{\mathrm{rad}}
$$




$$
\begin{aligned}
& \mathcal{M}_{S, 12}^{\prime 2}=\sin 2 \beta\left(\cos 2 \beta\left(M_{Z}^{2}-\lambda^{2} v^{2}\right)-\frac{1}{2} \Delta_{\mathrm{rad}}\right) \\
& \mathcal{M}_{S, 13}^{\prime 2}=\lambda v\left(2 \mu-\left(A_{\lambda}+2 \nu\right) \sin 2 \beta\right) \\
& \mathcal{M}_{S, 22}^{\prime 2}=M_{A}^{2}+\left(M_{Z}^{2}-\lambda^{2} v^{2}\right) \sin ^{2} 2 \beta+\cos ^{2} \beta \Delta_{\mathrm{rad}} \\
& \mathcal{M}_{S, 23}^{\prime 2}=\lambda v\left(A_{\lambda}+2 \nu\right) \cos 2 \beta \\
& \mathcal{M}_{S, 33}^{\prime 2}=\lambda A_{\lambda} \frac{v^{2}}{2 s} \sin 2 \beta+\nu\left(A_{\kappa}+4 \nu\right)
\end{aligned}
$$

where we have defined $\nu=\kappa s$ and

$$
M_{A}^{2}=\frac{2 \mu}{\sin 2 \beta}\left(A_{\lambda}+\nu\right),
$$

the mass squared of the MSSM-like CP-odd state $A$. ( $A$ mixes, in principle, with a mostly singlet-like state $A_{S}$. We will comment on the mass range of the CP-odd states in section 4.3.)

After an additional final diagonalisation the eigenstates will be denoted as

- $H_{S M}$ (dominantly SM-like)

- $H_{S}$ (dominantly singlet-like) and

- $H$ (dominantly the MSSM-like heavy scalar).

By this final diagonalisation the state $H_{S}$ picks up couplings to electroweak gauge bosons (vector bosons) proportional to the $H_{S M}^{\prime}-S^{\prime}$ mixing angle. Defining by $\kappa_{V}$ the ratio of couplings of a Higgs state to vector bosons relative to the corresponding coupling of the SM-like Higgs boson, one has

$$
\kappa_{V}^{2}\left(H_{S M}\right)+\kappa_{V}^{2}\left(H_{S}\right)+\kappa_{V}^{2}(H)=1 .
$$

$H_{S M}^{\prime}-S^{\prime}$ mixing will necessarily generate $\kappa_{V}^{2}\left(H_{S}\right) \neq 0$ and hence reduce $\kappa_{V}^{2}\left(H_{S M}\right)$, which is already [3] and will be even more constrained by Higgs coupling measurements at the LHC. Similarly, the state $H_{S}$ picks up couplings to fermions by both $H_{S M}^{\prime}-S^{\prime}$ and $H^{\prime}-S^{\prime}$ mixing, leading to non-vanishing values for $\kappa_{U}\left(H_{S}\right)$ (the reduced coupling of $H_{S}$ to uptype quarks) and $\kappa_{D}\left(H_{S}\right)$ (the reduced coupling of $H_{S}$ to down-type quarks). Then loop diagrams generate non-vanishing values for $\kappa_{g g}\left(H_{S}\right)$ (the reduced coupling of $H_{S}$ to gluons) and $\kappa_{\gamma \gamma}\left(H_{S}\right)$ (the reduced coupling of $H_{S}$ to diphotons). It is important to note that the coupling of $H_{S}$ to down-type quarks can suffer from cancellations among the contributions from $H_{S M}^{\prime}-S^{\prime}$ and $H^{\prime}-S^{\prime}$ mixing, respectively [65]. This can result in a reduced branching fraction $B R\left(H_{S} \rightarrow b \bar{b}\right)$. Since this decay constitutes the dominant contribution to the total width of $H_{S}$, its reduction implies enhanced branching fractions into other final states like $\gamma \gamma$. It is thus not astonishing that the $B R\left(H_{S} \rightarrow \gamma \gamma\right)$ can be larger than the one of a SM-Higgs boson of corresponding mass, leading to $\kappa_{\gamma \gamma}\left(H_{S}\right)>1$.

The diagonal term in (2.10) associated with the mass of the mostly SM Higgs is

$$
\mathcal{M}_{S, 11}^{\prime 2}=M_{Z}^{2} \cos ^{2} 2 \beta+\lambda^{2} v^{2} \sin ^{2} 2 \beta+\sin ^{2} \beta \Delta_{\mathrm{rad}}
$$


where the first term is the tree level upper bound for the Higgs mass in the MSSM. Due to the wide mass gap between $M_{Z}$ and $\sim 125 \mathrm{GeV}$ it is necessary to consider mechanisms able to uplift the Higgs mass from its MSSM-like tree level value. In the MSSM this may be achieved by sizeable radiative corrections $\Delta_{\text {rad }}$ which require large $(\gg 1 \mathrm{TeV})$ values for at least one soft SUSY breaking stop mass term and/or $A_{t}$. Such soft SUSY breaking terms generate, via loop effects, a soft SUSY breaking Higgs mass term $m_{H_{u}}^{2}(<0)$ of the same order. On the other hand, combining the (tree level) minimisation equations of the potential for the vevs $v_{u}$ and $v_{d}$, one obtains

$$
M_{Z}^{2}=\frac{2\left(m_{H_{d}}^{2}-m_{H_{u}}^{2} \tan ^{2} \beta\right)}{\tan ^{2} \beta-1}-2 \mu^{2} .
$$

In the absence of fine tuning, no large cancellations between the terms on the right hand side should occur. Hence large radiative corrections $\Delta_{\text {rad }}$ generate a so-called "little fine tuning problem" in the MSSM [12-16]. Moreover, the (effective) $\mu$ parameter should not be much larger than $M_{Z}$.

The second term in (2.13) is the well known NMSSM-specific contribution to the SMlike Higgs mass [17, 18], which is numerically relevant for $\tan \beta \lesssim 6$ and large $\lambda$. Avoiding a Landau singularity below the GUT scale requires $\lambda \lesssim 0.75$, limiting the possible uplift of the mass of the SM-like Higgs state to $\lesssim 17 \mathrm{GeV}$.

A third possibility to uplift the mass of the SM-like Higgs state has recently been studied in some detail in $[45,52]$ : if the diagonal term $\mathcal{M}_{S, 33}^{\prime 2}$ in (2.10) associated with the mass of the singlet-like Higgs state $S^{\prime}$ is smaller than $\mathcal{M}_{S, 11}^{\prime 2}, H_{S M}^{\prime}-S^{\prime}$ mixing induced by the term $\mathcal{M}_{S, 13}^{\prime 2}$ in (2.10) shifts upwards the mass of the SM-like Higgs state $H_{S M}$. The dominant contribution to $\mathcal{M}_{S, 13}^{\prime 2}$ originates from the first term $2 \lambda v \mu$, which gets reduced by the second term $-\lambda v\left(A_{\lambda}+2 \nu\right) \sin 2 \beta$. This reduction becomes small for moderate to large values of $\tan \beta[45,52]$. On the other hand, $H_{S M}^{\prime}-S^{\prime}$ mixing induces couplings of the lighter eigenstate $H_{S}$ to electroweak gauge bosons, $b \bar{b}$ and gluons (through top quark loops). Such couplings of a state with a mass below $114 \mathrm{GeV}$ are constrained by LEP [59]. This limits the region of $\lambda$ for a sizeable uplift the mass of the SM-like Higgs state to $\lambda \sim 0.04 \ldots 0.1$, and the possible uplift the mass of the SM-like Higgs state to $\lesssim 8 \mathrm{GeV}[45,52]$.

Subsequently we intend to quantify the NMSSM-specific uplifts of the mass of the SM-like Higgs state. To this end we define a mass shift $\Delta_{\text {NMSSM }}$ of the mostly SM-like Higgs state due to the NMSSM specific effects, from the second term in (2.13) and/or from $H_{S M}^{\prime}-S^{\prime}$ mixing. Contributions from $H_{S M}^{\prime}-S^{\prime}$ mixing are easy to identify; it suffices to compare the second eigenvalue of $\mathcal{M}_{S}^{2}$ (corresponding to $M_{H_{S M}}^{2}$ ) to the case where $\lambda, \kappa \rightarrow 0$ (keeping $\mu$ fixed, which requires to keep the ratio $\kappa / \lambda$ fixed). Such a definition of $\Delta_{\text {NMSSM }}$ has already been employed in $[45,52]$. In addition we want to keep track of the NMSSM contribution from the second term in (2.13) relative to the MSSM, which is relevant for small $\tan \beta$ only. But keeping small $\tan \beta$ would reduce the MSSM-like tree level value $M_{Z}^{2} \cos ^{2} 2 \beta$, and it would not be "fair" to compare the NMSSM to the MSSM for low values of $\tan \beta$. Hence we evaluate the contribution to $\Delta_{\text {NMSSM }}$ in the large $\lambda$-low $\tan \beta$ regime of the NMSSM by comparing to the $\operatorname{MSSM}(\lambda, \kappa \rightarrow 0$ as before) with a large value of $\tan \beta=40$. (The SM-like Higgs mass in the MSSM is practically independent of 
$\tan \beta$ for $\tan \beta>40$.) Therefore, for a given set of parameters in (2.6),

$$
\Delta_{\mathrm{NMSSM}}=M_{H_{S M}}-\left.\max _{\tan \beta} M_{H_{S M}}\right|_{\lambda, \kappa \rightarrow 0} \simeq M_{H_{S M}}-\left.M_{H_{S M}}\right|_{\lambda, \kappa \rightarrow 0, \tan \beta=40} .
$$

Clearly, larger values of $\Delta_{\text {NMSSM }}$ require smaller radiative corrections $\Delta_{\text {rad }}$ to $\mathcal{M}_{S, 11}^{\prime 2}$ and alleviate correspondingly the little hierarchy problem. Accordingly $\Delta_{\mathrm{NMSSM}}$ can be interpreted as an approximate measure of naturalness.

It is the aim of the present paper to study in how far such natural regions in the parameter space of the NMSSM can be tested in the future, as function of $\Delta_{\text {NMSSM }}$ and the mechanism for an NMSSM-specific uplift of the mass of the SM-like Higgs state. Since $H_{S M}^{\prime}-S^{\prime}$ mixing has a negative effect on $\Delta_{\mathrm{NMSSM}}$ for $M_{H_{S}}>125 \mathrm{GeV}$ (also if $\Delta_{\mathrm{NMSSM}}$ originates mainly from the second term in (2.13)) we will concentrate on $M_{H_{S}}<125 \mathrm{GeV}$. Then, present constraints and future discoveries/constraints can originate from

- direct searches for $H_{S}$ in the diphoton final state, which had been carried out by ATLAS for $65 \mathrm{GeV}<M_{H_{S}}[62]$ and by CMS for $80 \mathrm{GeV}<M_{H_{S}}<115 \mathrm{GeV}$ [63].

- measurements of the reduced signal rates/couplings (with respect to the SM) of $H_{S M}$. In the case of $H_{S M}^{\prime}-S^{\prime}$ mixing, these signal rates/couplings diminish proportional to the mixing angle.

- possible production of $H_{S}$ in decays of the MSSM-like states $H / A$.

Comparing the corresponding sensitivities allows to verify under which conditions natural NMSSM scenarios with $M_{H_{S}}<125 \mathrm{GeV}$ can be tested at future runs at the LHC, depending on the mechanism for the NMSSM-specific uplift of the mass of the SM-like Higgs state. To this end we have scanned the parameter space of the NMSSM as described in the next section.

\section{Numerical analysis}

We have performed these calculations with the public code NMSSMTools_4.4.0 $[76,77]$ including up to two-loop radiative corrections to the Higgs mass matrices as obtained in [78]. All phenomenological constraints, including the absence of Landau singularities below the GUT scale and, notably, constraints from Higgs searches in various channels at LEP are applied as in NMSSMTools (except for $(g-2)_{\mu}$ ).

The NMSSM specific parameters in eq. (2.13) are varied in the ranges

$$
\begin{aligned}
& 0.001 \leq \lambda<0.75, \quad 0.001 \leq \kappa \leq 0.75, \quad 1 \leq \tan \beta \leq 50, \\
& 0 \leq A_{\lambda} \leq 2.5 \mathrm{TeV}, \quad-1 \mathrm{TeV} \leq A_{\kappa} \leq 0, \quad 100 \mathrm{GeV} \leq \mu \leq 250 \mathrm{GeV} ;
\end{aligned}
$$

we found that wider ranges of the trilinear couplings $A_{\lambda}, A_{\kappa}$ and $\mu$ (including negative values of $\kappa$ and/or $\mu$ ) have practically no impact on our results. The soft SUSY breaking squark masses of the third generation $M_{U_{3}}, M_{D_{3}}, M_{Q_{3}}$ and the stop mixing parameter $A_{t}$ are confined to ranges below $1 \mathrm{TeV}$ in order to avoid too large fine tuning:

$$
700 \mathrm{GeV} \leq M_{U_{3}}=M_{D_{3}}=M_{Q_{3}} \leq 1 \mathrm{TeV}, \quad-1 \mathrm{TeV} \leq A_{t} \leq 1 \mathrm{TeV} .
$$


(For $\left|A_{t}\right| \leq 1 \mathrm{TeV}$, third generation squark masses below $\sim 700 \mathrm{GeV}$ do not allow to reach $125.1 \pm 3 \mathrm{GeV}$ for $M_{H_{S M}}$ even in the NMSSM.) The lightest physical stop mass $m_{\tilde{t}_{1}}$ satisfies $m_{\tilde{t}_{1}} \gtrsim 480 \mathrm{GeV}$.

The soft SUSY breaking mass terms and trilinear couplings for the sleptons have been set to $500 \mathrm{GeV}$ and $550 \mathrm{GeV}$ respectively, whereas for the squarks of first two generations the masses are set to $2 \mathrm{TeV}$. The gluino mass is chosen as $M_{3}=1.6 \mathrm{TeV}$, and the other soft SUSY breaking gaugino masses such that they satisfy approximately universal relations at the GUT scale, i.e. $M_{2}=2 M_{1}=M_{3} / 3$. (All these parameters have practically no impact on our results.)

For each point in the parameter space satisfying the phenomenological constraints, including a SM-like Higgs state with a mass of $125.1 \pm 3 \mathrm{GeV}$ (allowing for theoretical uncertainties) and couplings of $H_{S M}$ to gauge bosons and fermions in the 95\% CL ranges given in [3, 70], we further require $M_{H_{s}}<M_{H_{S M}}$. Then we compute for each point $\Delta_{\mathrm{NMSSM}}$ according to the procedure described above, and various observables like reduced couplings and signal rates for the relevant Higgs states shown in the next section.

\section{Results}

Due to the limited range (3.2) for the soft SUSY breaking squark masses of the third generation and the stop mixing parameter, all viable points need a non-vanishing value of $\Delta_{\text {NMSSM }}$ in the range $4 \mathrm{GeV} \lesssim \Delta_{\text {NMSSM }} \lesssim 17 \mathrm{GeV}$ in order reach a SM-like Higgs mass of $125.1 \pm 3 \mathrm{GeV}$. Hence this range for the soft SUSY breaking squark masses of the third generation and the stop mixing parameter, motivated by alleviating the little hierarchy problem, is not viable in the MSSM.

Turning to the possible mechanisms for an uplift of the mass of the SM-like Higgs state, it follows from the discussion in section 2 that these take place in different regions of $\lambda$ and $\tan \beta$ : contributions to $\Delta_{\mathrm{NMSSM}}$ up to $\sim 17 \mathrm{GeV}$ from the second term in (2.13) (limited by the absence of a Landau singularity of $\lambda$ below the GUT scale) are possible for large $\lambda$ and $\tan \beta \lesssim 6$; subsequently this region will be denoted as "large $\lambda$ " (LLAM) region. The region where contributions to $\Delta_{\text {NMSSM }}$ from $H_{S M}^{\prime}-S^{\prime}$ mixing are sizeable (up to $\sim 8 \mathrm{GeV}$ ) is characterised by a small value of $\lambda$ and large $\tan \beta$. Subsequently we call this region the "large mixing" (LMIX) region.

The viable points are shown in the $\lambda-\tan \beta$ plane in figure 1 , including the possible values of $\Delta_{\text {NMSSM }}$ in the form of a color code. One can clearly distinguish the two "islands" of valid points in the plane which can lead to a substantially different phenomenology, but both featuring a lower fine tuning than in the MSSM. In the following subsections we show various observables which can help to test these scenarios.

\subsection{Searches for $H_{S}$ in the diphoton final state}

As already stated above, the ATLAS and CMS collaborations have recently published results from searches for additional BSM Higgs bosons with masses below $125 \mathrm{GeV}$ in the diphoton final state $[62,63]$, leading to upper bounds on corresponding signal rates. First we have to verify whether these upper bounds lead to constraints on the parameter space 


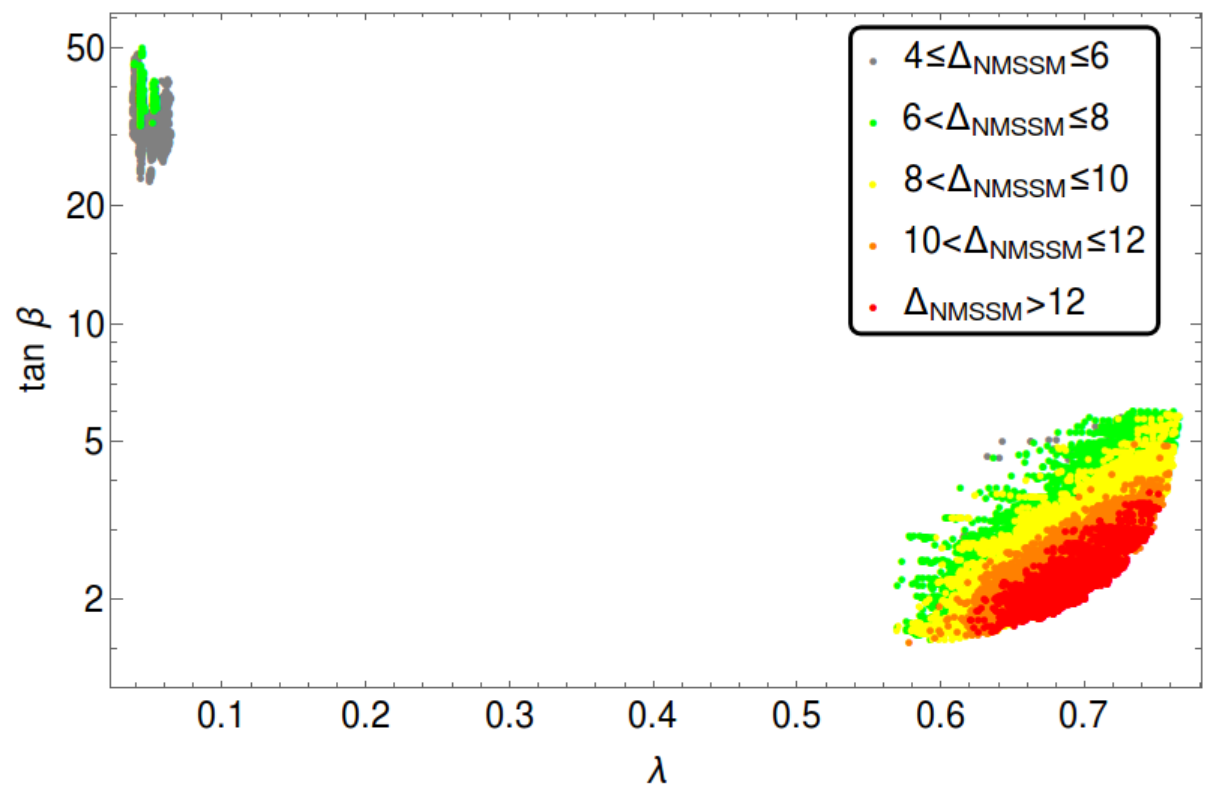

Figure 1. $\lambda-\tan \beta$ plane showing the viable points and $\Delta_{\text {NMSSM }}$ in the form of a color code. The island in the upper-left corner corresponds to the region where $\Delta_{\mathrm{NMSSM}}$ originates from $H_{S M}^{\prime}-S^{\prime}$ mixing (LMIX), whereas the island in the large $\lambda$ regime (LLAM) corresponds to the region with large contributions to $\Delta_{\mathrm{NMSSM}}$ from the second term in (2.13).

of the NMSSM considered above. To this end we have used the public code SusHi 1.5 [79] to obtain the NNLO gluon fusion production cross section for a SM-like Higgs boson, and multiplied it by the reduced coupling of $H_{S}$ to gluons $\kappa_{g g}^{2}\left(H_{S}\right)$ given by the output of NMSSMTools. Finally the production cross section is multiplied by the $B R\left(H_{S} \rightarrow \gamma \gamma\right)$ as given by NMSSMTools.

On the left hand side of figure 2 we show the resulting signal rates at $\sqrt{s}=8 \mathrm{TeV}$ c.m. energy, together with the ATLAS [62] and CMS [63] limits from direct searches as function of $M_{H_{S}}$. Here the LMIX region apprears as a grey-green island within the much larger LLAM region. On the right hand side of figure 2 we show the resulting signal rates at $\sqrt{s}=13 \mathrm{TeV}$ c.m. energy, once the constraints from ATLAS and CMS searches have been applied.

We see in figures 2 that in the grey-green LMIX region $M_{H_{S}}$ is confined to the mass range $88 \mathrm{GeV} \lesssim M_{H_{S}} \lesssim 102 \mathrm{GeV}$, a consequence of the parameter range (3.2) and the corresponding lower limit on $\Delta_{\mathrm{NMSSM}} \gtrsim 4 \mathrm{GeV}$. In order to obtain such values of $\Delta_{\mathrm{NMSSM}}$ through $H_{S M}^{\prime}-S^{\prime}$ mixing, the mixing angle has to be relatively large leading to sizeable couplings of $H_{S}$ to electroweak gauge bosons. These, in turn, are allowed by LEP only in the corresponding mass range where, actually, a mild excess of events is seen [59].

The recent ATLAS and CMS searches have not yet been sensitive to the possible signal rates $\sigma\left(g g \rightarrow H_{S} \rightarrow \gamma \gamma\right)$ in the LMIX region of the NMSSM, due to the absence of a possible enhancement of the $B R\left(H_{S} \rightarrow \gamma \gamma\right)$ (see below). Figure 2 (right) indicates, on the other hand, that the LMIX region could be completely tested once searches at $\sqrt{s}=13 \mathrm{TeV}$ c.m. energy become sensitive to $\sigma\left(g g \rightarrow H_{S} \rightarrow \gamma \gamma\right) \sim 20 \mathrm{fb}$. 

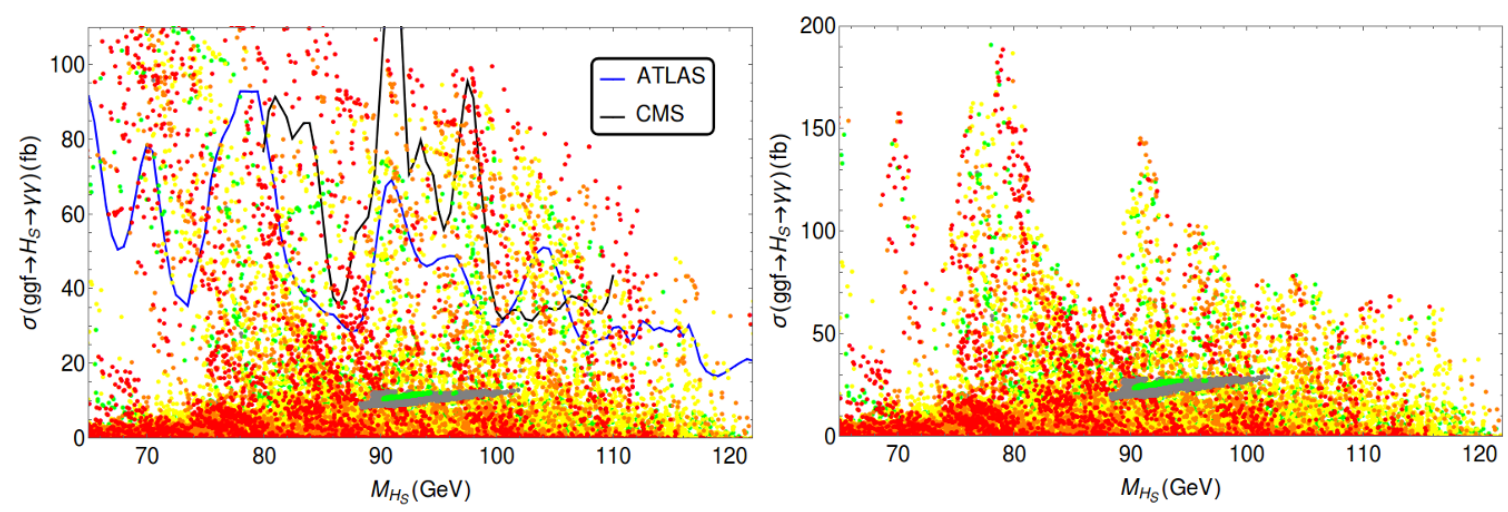

Figure 2. Left: possible signal rates (in femtobarns) $\sigma\left(g g \rightarrow H_{S} \rightarrow \gamma \gamma\right.$ ) at a c.m. energy of $\sqrt{s}=8 \mathrm{TeV}$, together with the ATLAS [62] and CMS [63] limits from direct searches. The greygreen island corresponds to the LMIX region, the rest to the LLAM region. Right: signal rates for the same process at $\sqrt{s}=13 \mathrm{TeV}$ for the remaining points once the upper bounds from ATLAS and CMS have been applied.

Within the LLAM (large $\lambda$ ) region both $M_{H_{S}}$ and $\sigma\left(g g \rightarrow H_{S} \rightarrow \gamma \gamma\right)$ can vary over much larger ranges and, indeed, the ATLAS and CMS searches have started to test parts of the LLAM region where this signal rate is particularly large. On the other hand this signal rate can also be quite small in the LLAM region where $H_{S M}^{\prime}-S^{\prime}$ mixing is possible, but not mandatory. This part of the LLAM region will be hard to test via searches for direct $H_{S}$ production.

It is interesting to decompose $\sigma\left(g g \rightarrow H_{S} \rightarrow \gamma \gamma\right)$ into production cross sections and branching fractions, which allows to estimate signal rates in other channels and to understand the origin of the varying signal rates in figure 2 . In figure 3 we show the production cross section of $H_{S}$ at $\sqrt{s}=8 \mathrm{TeV}$ (left) and $\sqrt{s}=13 \mathrm{TeV}$ (right) with the same color code for $\Delta_{\text {NMSSM }}$ as in figure 1 , omitting the points excluded by ATLAS or CMS. We observe that, for the allowed mass range $88 \mathrm{GeV} \lesssim M_{H_{S}} \lesssim 102 \mathrm{GeV}, \sigma\left(g g \rightarrow H_{S}\right)$ is indeed larger in the LMIX region than in the LLAM region, since the couplings of $H_{S}$ to fermions (here: to the top quark) are relatively large. However, the $B R\left(H_{S} \rightarrow \gamma \gamma\right)$ shown on the left hand side of figure 4 clarify that these can be (much!) larger for $H_{S}$ than for a SM-like Higgs (shown as blue line) only for parts of the LLAM region, never within the LMIX region; only within the LLAM region a suppression of the $B R\left(H_{S} \rightarrow b \bar{b}\right.$ ) is possible (as shown on the right hand side of figure 4) which is required in order to enhance the $B R\left(H_{S} \rightarrow \gamma \gamma\right)$.

Finally both figures 3 and 4 show that very few viable points exist for $M_{H_{S}}<60 \mathrm{GeV}$ (in the LLAM region only): such light states can be produced in decays $H_{S M} \rightarrow H_{S} H_{S}$ and would reduce the observed $H_{S M}$ signal rates into SM-like final states to inadmissible levels. The $H_{S M}-H_{S}-H_{S}$ coupling can be small for large $\lambda$, however, due to (rare) accidential cancellations among the various contributing terms. (This mass range has not been shown in figure 2 since the experiments have not been sensitive to it.) 

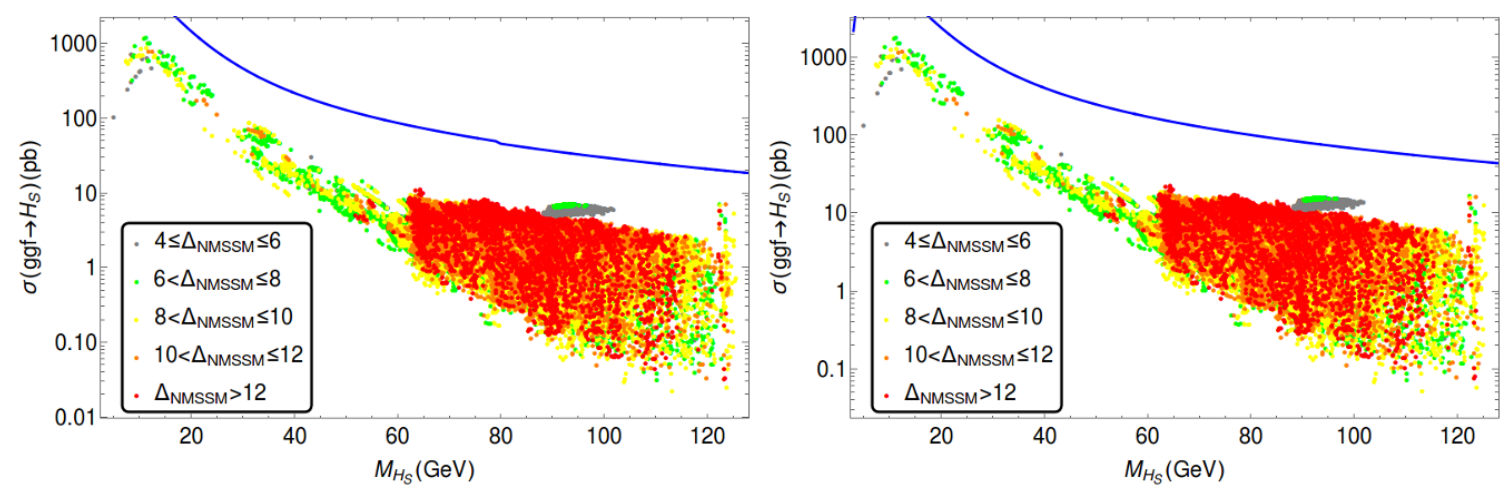

Figure 3. Production cross section of $H_{S}$ at $\sqrt{s}=8 \mathrm{TeV}$ (left) and $\sqrt{s}=13 \mathrm{TeV}$ (right) with the color code for $\Delta_{\text {NMSSM }}$. The blue line indicates the corresponding ggF cross section for a SM Higgs boson of the same mass. The grey-green island corresponds to the LMIX region.
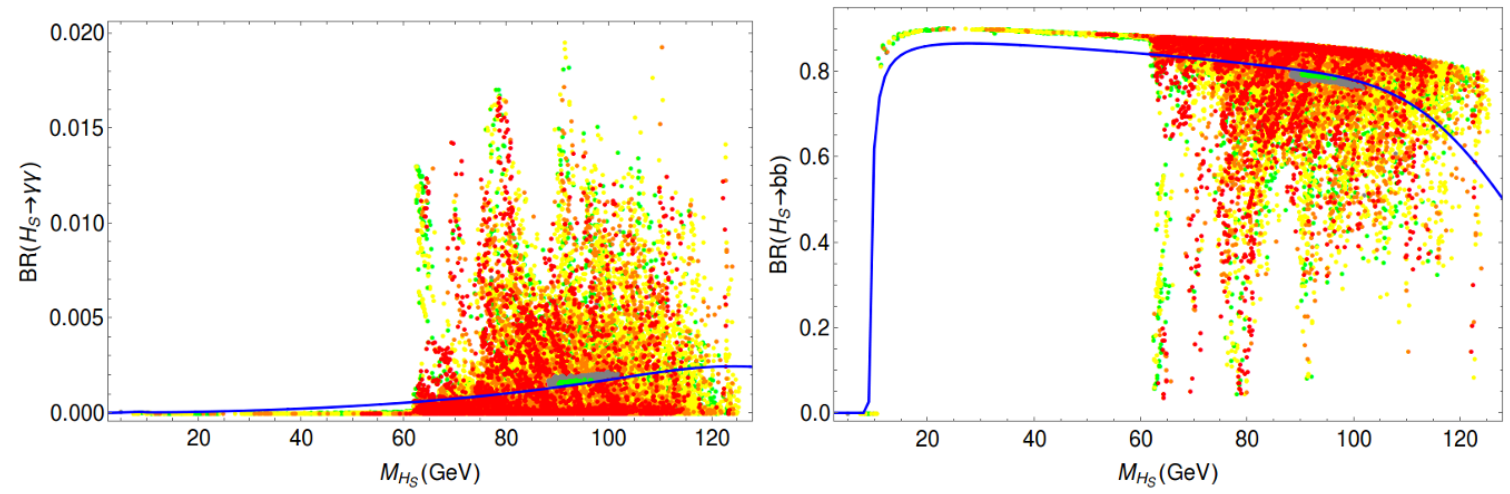

Figure 4. Branching ratios of $H_{S}$ into photons (left) and $b \bar{b}$ (right) versus its mass. The blue line indicates the corresponding branching ratios for a SM Higgs boson of the same mass. The grey-green island corresponds to the LMIX region, in which the branching ratios are very SM-like.

\subsection{Reduced couplings of $H_{S M}$}

As stated above the LMIX (and LLAM) regions can have an impact on the reduced couplings of $H_{S M}$, actually both due to $H_{S M}^{\prime}-S^{\prime}$ mixing and $H_{S M}^{\prime}-H^{\prime}$ mixing induced by the final diagonalisation of the mass matrix $\mathcal{M}^{\prime 2}(2.10)$. The ATLAS and CMS measurements of the reduced couplings of $H_{S M}$ at the first run of the LHC have recently been combined in [3], and prospects for future measurements have been published in [60] (ATLAS) and [61] (CMS).

First we show in figure 5 the reduced couplings $\kappa_{V}\left(H_{S M}\right)$ and $\kappa_{\gamma \gamma}\left(H_{S M}\right)$ for the viable points. The LMIX and LLAM regions can be distinguished clearly in figure 5: as before the LMIX region corresponds to the thin grey-green strip, the LLAM region to the remaining part dominated by mostly red points (for which $12 \mathrm{GeV}<\Delta_{\text {NMSSM }}<17 \mathrm{GeV}$ ).

From the recent ATLAS-CMS combination in [3] one finds for the scenario relevant here (custodial symmetry, i.e. $\left.\kappa_{Z}\left(H_{S M}\right)=\kappa_{W}\left(H_{S M}\right) \equiv \kappa_{V}\left(H_{S M}\right) \leq 1\right)$ that $\kappa_{V}\left(H_{S M}\right) \gtrsim 0.83$ at the $95 \%$ CL level. The prospects for the measurements of $\kappa_{V}\left(H_{S M}\right)$ at the run II of the LHC in [60] (ATLAS) and [61] (CMS) depend on uncertainty scenarios and, of course, 


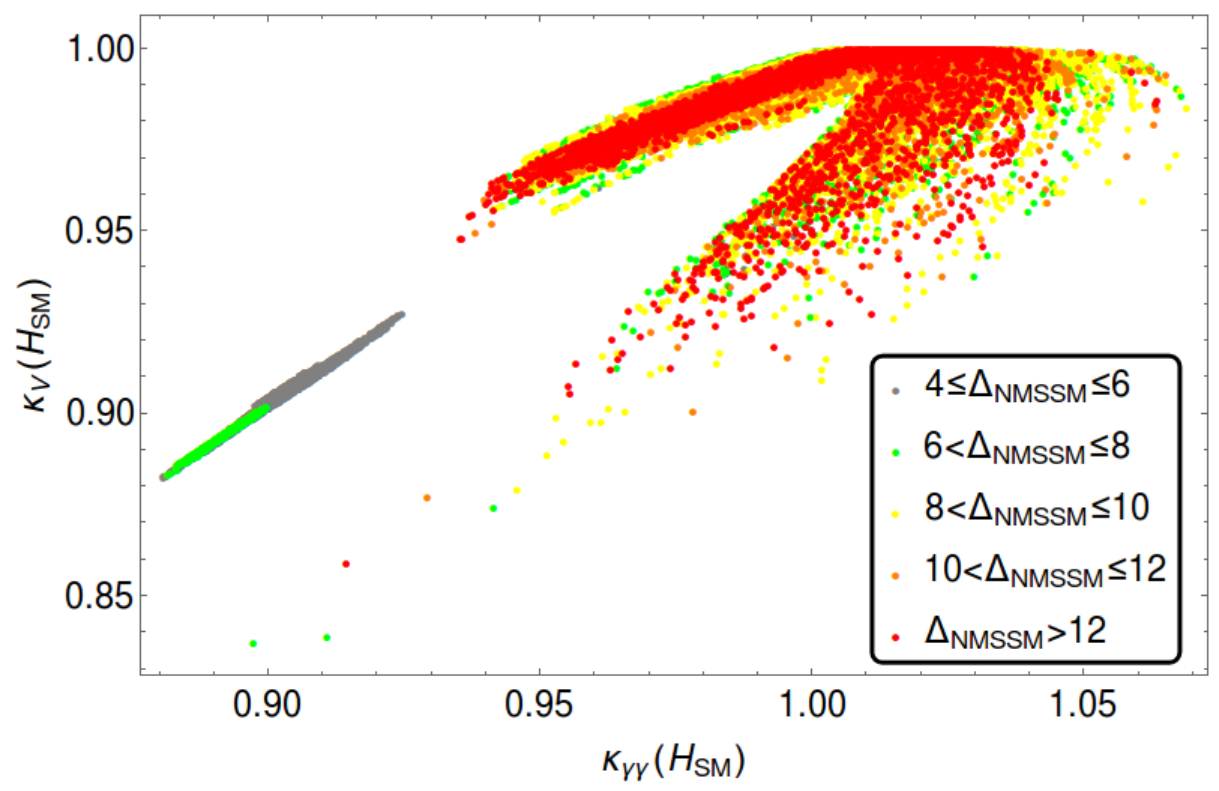

Figure 5. Reduced couplings $\kappa_{V}\left(H_{S M}\right)$ and $\kappa_{\gamma \gamma}\left(H_{S M}\right)$ for the viable points, including a color code for $\Delta_{\mathrm{NMSSM}}$.

on the integrated luminosity. For $300 \mathrm{fb}^{-1}$ one expects uncertainties of about $5 \%$ at the $1 \sigma$ level, i.e. the possibility to set a lower bound on $\kappa_{V}\left(H_{S M}\right)$ of $\sim 0.9$ at the $95 \%$ CL level. Such a bound can test the green $\Delta_{\mathrm{NMSSM}}>6 \mathrm{GeV}$ region of the LMIX scenario, but reduced uncertainties of about $7 \%$ at the $95 \%$ CL level at $3000 \mathrm{fb}^{-1}$ integrated luminosity could test the LMIX scenario completely. Again, the LLAM scenario can be tested only partially by measurements of $\kappa\left(H_{S M}\right)$. The prospects for constraining (or detecting) the LMIX/LLAM scenarios via measurements of $\kappa_{\gamma \gamma}\left(H_{S M}\right)$ are similar, but somewhat less promising due to the larger foreseen uncertainties at both $300 \mathrm{fb}^{-1}$ and $3000 \mathrm{fb}^{-1}$ integrated luminosity [60,61].

Apart by future measurements of individual values of reduced couplings of $H_{S M}$, informations or constraints on scenarios predicting deviations from the SM can be obtained by considering correlations among reduced couplings. To this end we show in figures 6 the correlations of $\kappa_{V}\left(H_{S M}\right)$ with the reduced couplings of $H_{S M}$ to down-type fermions $\left(\kappa_{D}\left(H_{S M}\right)\right)$ and gluons $\left(\kappa_{g g}\left(H_{S M}\right)\right)$.

Like in figure 5 these correlations are very pronounced in the LMIX scenario, but in the LLAM scenario a wide range of $\kappa_{D}\left(H_{S M}\right)$ is possible: a reduction of the coupling of $H_{S M}$ to down-type fermions originates from negative contributions to this coupling from $H_{S M}^{\prime}-H^{\prime}$ mixing. As for $H_{S}$, a corresponding reduction of the $B R\left(H_{S M} \rightarrow b \bar{b}\right)$ can lead to an enhanced $B R\left(H_{S M} \rightarrow \gamma \gamma\right)$ as observed in figure 5. However, positive contributions to the coupling of $H_{S M}$ to down-type fermions are possible as well, with opposite consequences. The two regions $\kappa_{D}\left(H_{S M}\right)>1$ and $\kappa_{D}\left(H_{S M}\right)<1$ explain the origin of the two "branches" of $\kappa_{V}\left(H_{S M}\right)$ visible in figure 5 as well on the right hand side of figure 6. Unfortunately, the couplings of $H_{S M}$ can also be very SM-like, like in the alignment limit studied recently in [80]. 

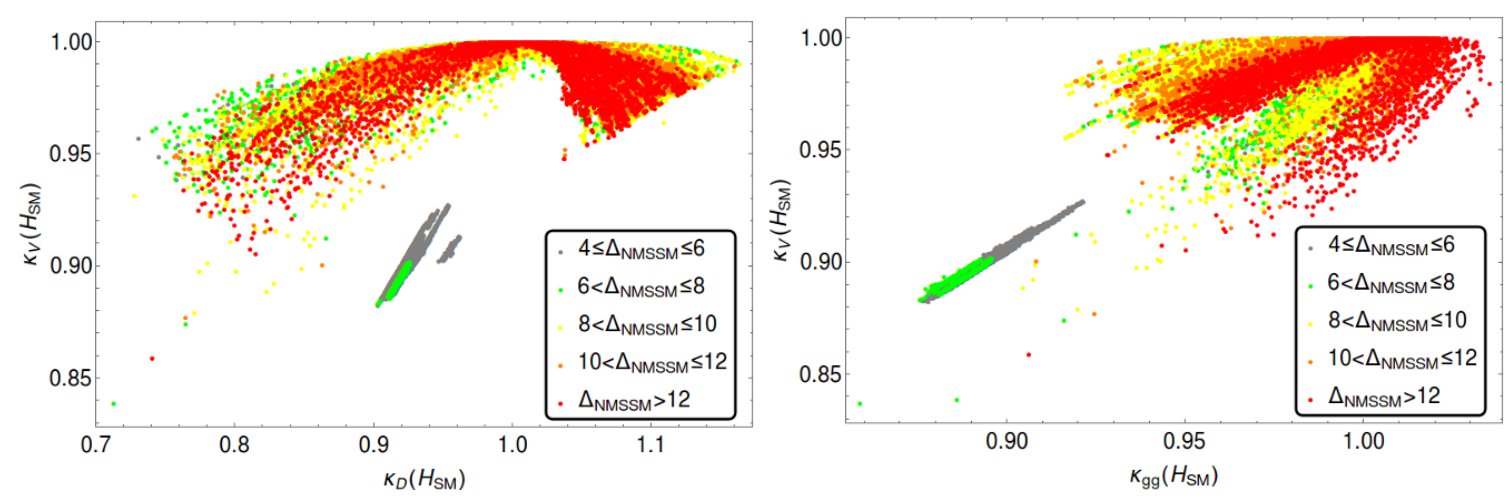

Figure 6. Correlations of $\kappa_{V}\left(H_{S M}\right)$ with the reduced couplings of $H_{S M}$ to down-type fermions (left) and gluons (right). In both plots the two regions LLAM and LMIX are clearly separated.
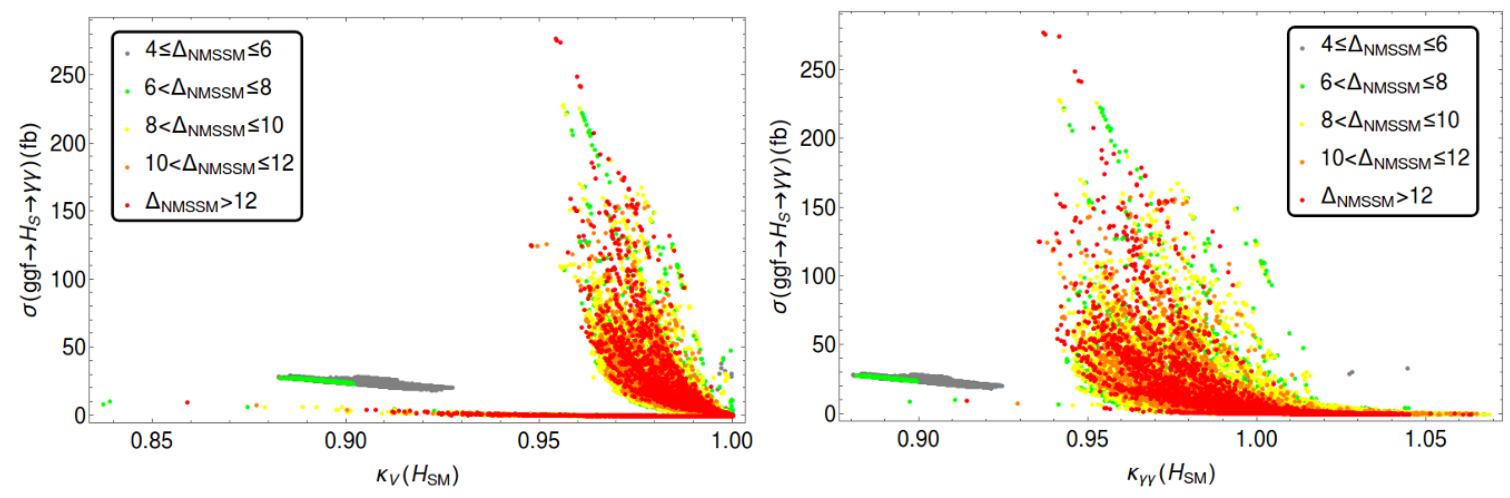

Figure 7. Left: correlations among the diphoton signal rate of $H_{S}$ and $\kappa_{V}\left(H_{S M}\right)$. Right: correlations among the diphoton signal rate of $H_{S}$ and $\kappa_{\gamma \gamma}\left(H_{S M}\right)$.

Next we turn to correlations between the reduced couplings of $H_{S M}$ and the signal rates $\sigma\left(g g \rightarrow H_{S} \rightarrow \gamma \gamma\right)$ discussed in the previous subsection. In figures 7 we show $\sigma\left(g g \rightarrow H_{S} \rightarrow \gamma \gamma\right)$ against $\kappa_{V}\left(H_{S M}\right)$ (left) and $\sigma\left(g g \rightarrow H_{S} \rightarrow \gamma \gamma\right)$ against $\kappa_{\gamma \gamma}\left(H_{S M}\right)$ (right). These figures allow to verify the possible complementarity of measurements of $\sigma\left(g g \rightarrow H_{S} \rightarrow \gamma \gamma\right)$ and the reduced couplings of $H_{S M}$ : in order to test the LMIX region (the grey-green island on the left hand side), the necessary limits on $\sigma\left(g g \rightarrow H_{S} \rightarrow \gamma \gamma\right)$ and/or $\kappa_{V}\left(H_{S M}\right)$ can now be deduced together. The LLAM region can become visible either by an enhanced $\sigma\left(g g \rightarrow H_{S} \rightarrow \gamma \gamma\right)$ or a reduced $\kappa_{V}\left(H_{S M}\right)$, but not both. Unfortunately, a low signal rate $\sigma\left(g g \rightarrow H_{S} \rightarrow \gamma \gamma\right)$ as well as $\kappa_{V}\left(H_{S M}\right) \sim 1$ are possible simultaneously. From the right hand side of figures 7 we see that enhanced signal rates $\sigma\left(g g \rightarrow H_{S} \rightarrow \gamma \gamma\right) \gtrsim 50 \mathrm{fb}$ and enhanced reduced couplings $\kappa_{\gamma \gamma}\left(H_{S M}\right)$ are incompatible in the LLAM region.

\section{3 $H_{S}$ production via decays of heavy states $H / A$}

Another way to produce a light $H_{S}$ is through the decays of heavy (MSSM-like) states $H / A$. First we have to find out which masses of $H / A$ are possible in the LMIX/LLAM regions of the NMSSM considered here. In figure 8 we show the regions of viable points in 


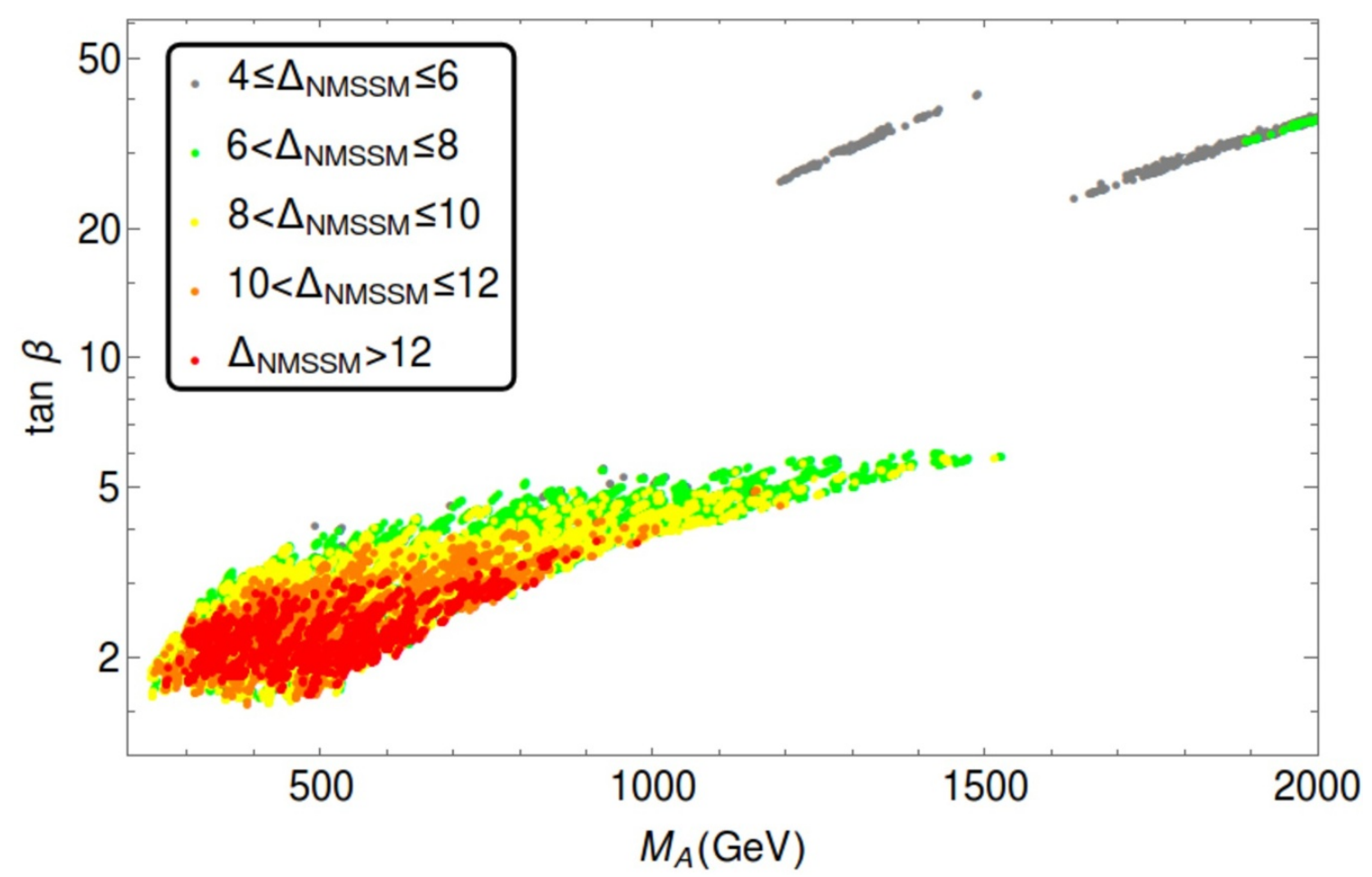

Figure 8. Viable points in the $\tan \beta-M_{A}$ plane.

the $\tan \beta-M_{A}$ plane, which helps to clarify that these points are not ruled out by searches for MSSM-like $H / A$ in the $\tau^{+} \tau^{-}$final state (from here onwards, $M_{A}$ denotes the physical mass of the MSSM-like CP-odd state $A$ ): the LMIX region with large $\tan \beta$ features very heavy $H / A$ states, to which searches at the run I have not been sensitive (and which will be hard to search for at the run II). The LLAM region is characterized by lower $\tan \beta$ such that the associate production of $H / A$ states with $b$ quarks is not very enhanced; instead, their production via gluon fusion becomes feasable in principle [81]. The part of the LLAM region where $M_{A} \gtrsim 500 \mathrm{GeV}$ and $\tan \beta \gtrsim 3$ corresponds, however, to the difficult region where the reduced couplings of $H_{S M}$ are very SM-like and $H_{S}$ has a low signal rate in the $\gamma \gamma$ channel; in this region also the search for the MSSM-like states $H / A$ seems difficult [82].

Promising decays of $H / A$ into $H_{S}$ are $A \rightarrow Z+H_{S}$ and $H \rightarrow H_{S M}+H_{S}$. Since the kinematics of $A \rightarrow Z+H_{S}$ is very similar to the one of $H \rightarrow Z+A_{S}$ investigated in [83], the studies of the $Z \rightarrow l^{+} l^{-}(l \equiv e, \mu)$ and $A_{S} \rightarrow b \bar{b}$ final states in [83] can be employed, including their sensitivity curves as function of $M_{A_{S}}$ (now interpreted as $M_{H_{S}}$ ). First we show what signal cross sections can be expected as function of $M_{A}$. The signal cross section $\sigma\left(g g F \rightarrow A \rightarrow Z+H_{S}\right)$ is shown on the left hand side of figure 9 as function of $M_{A}$; clearly, visible signal rates can only be expected for $M_{A} \lesssim 400 \mathrm{GeV}$ within the LLAM region. On the right hand side of figure 9 the range of signal cross sections $\sigma(g g F \rightarrow A \rightarrow Z+b+\bar{b})$ is shown as function of $M_{H_{S}}$, and compared to the expected sensitivities at the run II of the LHC for integrated luminosities of $300 \mathrm{fb}^{-1}$ (blue) and $3000 \mathrm{fb}^{-1}$ (black) (from [83]). Hence, detectable signal rates in this channel are indeed possible in the LLAM region of the NMSSM without, however, covering it completely. 

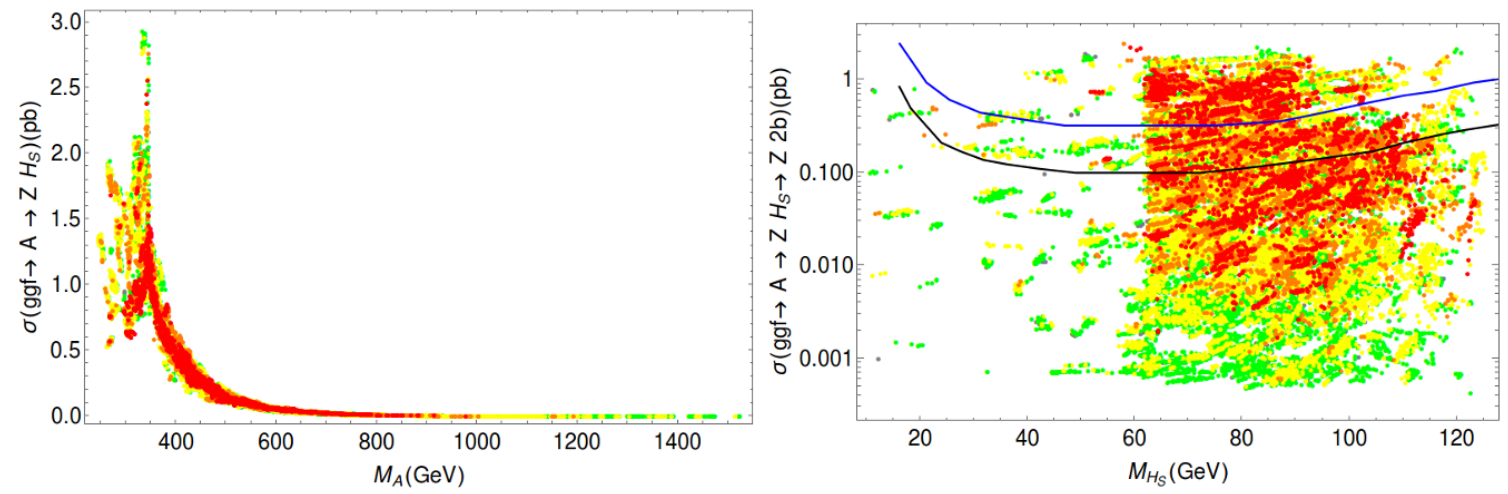

Figure 9. Left: signal cross section $\sigma\left(g g F \rightarrow A \rightarrow Z+H_{S}\right)$ as function of $M_{A}$ for a c.m. energy of $\sqrt{s}=13 \mathrm{TeV}$. Right: signal cross section $\sigma(g g F \rightarrow A \rightarrow Z+b+\bar{b})$ as function of $M_{H_{S}}$, compared to the expected sensitivities for a integrated luminosities of $300 \mathrm{fb}^{-1}$ (blue) and $3000 \mathrm{fb}^{-1}$ (black) (from [83]).
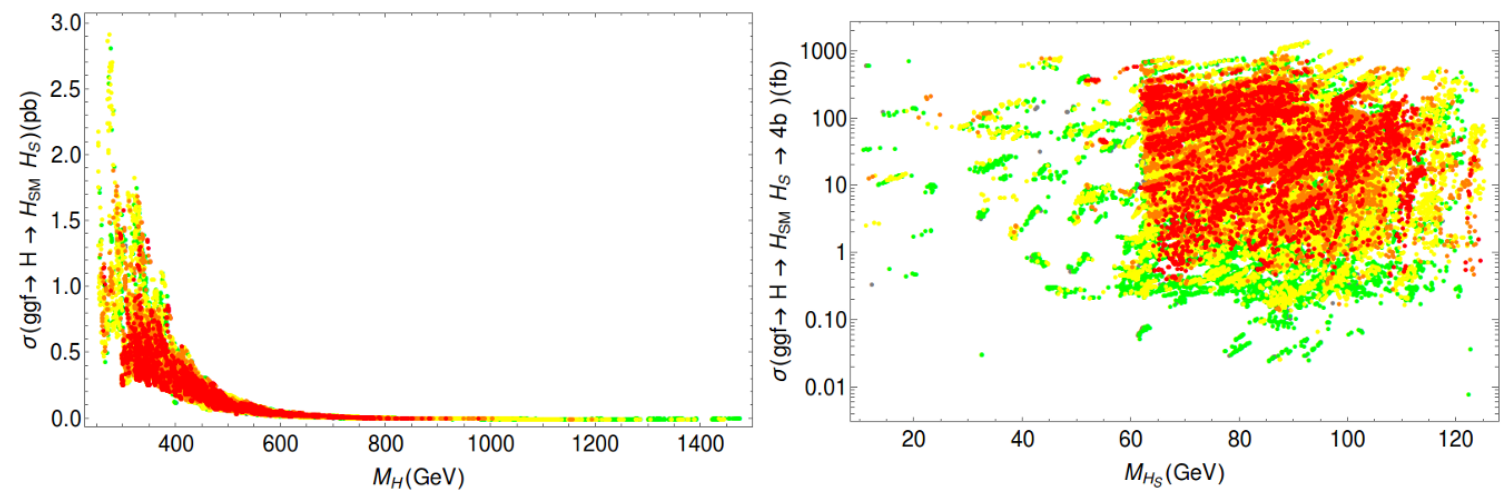

Figure 10. Total ggF production cross section for $H \rightarrow H_{S M}+H_{S}$ at a c.m. energy $\sqrt{s}=13 \mathrm{TeV}$ (left), and the signal cross section into $b \bar{b} b \bar{b}$ versus the mass of $H_{S}$ (right).

The process $H \rightarrow H_{S M}+H_{S}$ can, in principle, be searched for in various final states as $4 b, 2 b 2 \tau$ and $4 \tau$; one is handicapped, however, by the a priori unknown mass of $H_{S}$. In figure 10 we show the cross section $\sigma\left(g g F \rightarrow H \rightarrow H_{S M}+H_{S}\right)$ as function of $M_{H}$ for a c.m. energy of $\sqrt{s}=13 \mathrm{TeV}$ on the left, and the (dominant) signal cross section $\sigma\left(g g F \rightarrow H \rightarrow H_{S M}+H_{S} \rightarrow 4 b\right)$ as function of $M_{H_{S}}$ on the right. Search strategies including background studies can possibly be persued along the lines proposed in [83] for searches for a light NMSSM pseudoscalar $A_{S}$. In the region of the NMSSM parameter space considered here $A_{S}$ is, however, not particularly light; we found that, in the (wider) LLAM region, $M_{A_{S}}$ varies from $\sim 80$ to $\sim 300 \mathrm{GeV}$, but from $\sim 60$ to $\sim 180 \mathrm{GeV}$ in the (narrower) LMIX region. Search strategies including background studies for searches for $H_{S} / A_{S}$ in Higgs-to-Higgs decays are beyond the scope of the present paper and merit future studies. 


\section{Conclusions}

We have studied a region in the NMSSM parameter space in which the mass of the SM-like Higgs boson is uplifted by $\sim 4-17 \mathrm{GeV}$, allowing for both stop masses and $\left|A_{t}\right| \leq 1 \mathrm{TeV}$ alleviating the little fine tuning problem of the MSSM. This region features a lighter mostly singlet-like Higgs state $H_{S}$ with a mass in the $60-125 \mathrm{GeV}$ range if the uplift is due to singlet-doublet mixing (the LMIX region). Confining ourselves to values of $\lambda \lesssim 0.75$, this mass range of $H_{S}$ is also natural in the LLAM region where the uplift originates from the additional quartic term $\sim \lambda^{2}$ in the potential of the SM-like Higgs boson.

The aim of the paper is the study of possible direct or indirect searches for a light $H_{S}$ at the run II of the LHC. Three possibilities have been considered:

a) Direct production of $H_{S}$ in gluon fusion, with $H_{S}$ decaying into diphotons. Corresponding searches have been conducted recently by ATLAS and CMS (the results of which have been taken into account), and are the most promising also for the run II of the LHC.

b) Modified reduced couplings of the SM-like Higgs state $H_{S M}$ through singlet-doublet mixing (both in the LMIX and the LLAM regions).

c) Production of $H_{S}$ in decays of heavier $H / A$ states, where we confined ourselves to the most promising $A \rightarrow Z+H_{S}$ channel.

We found that the LMIX region can be tested if searches for BSM Higgs bosons in the mass range $88-102 \mathrm{GeV}$ become sensitive to signal cross sections $\sigma\left(g g \rightarrow H_{S} \rightarrow \gamma \gamma\right) \sim$ $20 \mathrm{fb}$. Alternatively, the LMIX region can be tested if measurements of the reduced coupling $\kappa_{V}\left(H_{S M}\right)$ of the SM Higgs boson to electroweak gauge boson exclude (or confirm) the region $\kappa_{V}\left(H_{S M}\right) \lesssim 0.93$. Since the $H / A$ states are always quite heavy in the LMIX region (with masses well above $1 \mathrm{TeV}), H_{S}$ detection via $H / A$ seems impossible in the near future, and tests of the LMIX region have to rely on one of the two measurements above, which seems feasable if the projected sensitivities can be reached.

On the other hand it is difficult to test the entire LLAM region even if $H_{S}$ is light (with a mass below $125 \mathrm{GeV}$ ), the range considered here: both the signal cross section $\sigma\left(g g \rightarrow H_{S} \rightarrow \gamma \gamma\right)$ and the deviation of the reduced couplings of $H_{S M}$ from one can simultaneously be very small. However, in other parts of the LLAM region both the signal cross section $\sigma\left(g g \rightarrow H_{S} \rightarrow \gamma \gamma\right)$ and the deviation of the reduced couplings of $H_{S M}$ from one can be much larger than in the LMIX region; these parts of the LLAM region will be the first ones to be tested. In a part of the "difficult" LLAM region, but for which the $H / A$ states are not too heavy (with masses $\lesssim 400 \mathrm{GeV}$ ), the detection of $H_{S}$ at least via $g g F \rightarrow A \rightarrow Z+H_{S}$ is possible. Studies on the possible detection of $H_{S}$ via other $H / A$ decay channels (including larger masses of $H_{S}$ ) are planned.

\section{Acknowledgments}

U.E. and M.R. acknowledge support from the European Union Initial Training Network HiggsTools (PITN-GA-2012-316704) and the Défi InPhyNiTi project N2P2M-SF. U.E. acknowledges support from European Union Initial Training Network INVISIBLES (PITN- 
GA-2011-289442), the ERC advanced grant Higgs@LHC, and from the grant H2020-MSCARISE-2014 No. 645722 (NonMinimalHiggs).

Open Access. This article is distributed under the terms of the Creative Commons Attribution License (CC-BY 4.0), which permits any use, distribution and reproduction in any medium, provided the original author(s) and source are credited.

\section{References}

[1] ATLAS collaboration, Observation of a new particle in the search for the Standard Model Higgs boson with the ATLAS detector at the LHC, Phys. Lett. B 716 (2012) 1 [arXiv: 1207.7214] [INSPIRE].

[2] CMS collaboration, Observation of a new boson at a mass of $125 \mathrm{GeV}$ with the CMS experiment at the LHC, Phys. Lett. B 716 (2012) 30 [arXiv:1207.7235] [INSPIRE].

[3] ATLAS and CMS collaborations, Measurements of the Higgs boson production and decay rates and constraints on its couplings from a combined ATLAS and CMS analysis of the LHC pp collision data at $\sqrt{s}=7$ and 8 TeV, ATLAS-CONF-2015-044, CERN, Geneva Switzerland (2015) [CMS-PAS-HIG-15-002].

[4] L.J. Hall, D. Pinner and J.T. Ruderman, A natural SUSY Higgs near 126 GeV, JHEP 04 (2012) 131 [arXiv:1112.2703] [INSPIRE].

[5] H. Baer, V. Barger and A. Mustafayev, Implications of a $125 \mathrm{GeV}$ Higgs scalar for LHC SUSY and neutralino dark matter searches, Phys. Rev. D 85 (2012) 075010 [arXiv: 1112.3017] [INSPIRE].

[6] S. Heinemeyer, O. Stål and G. Weiglein, Interpreting the LHC Higgs search results in the MSSM, Phys. Lett. B 710 (2012) 201 [arXiv:1112.3026] [INSPIRE].

[7] A. Arbey, M. Battaglia, A. Djouadi, F. Mahmoudi and J. Quevillon, Implications of a 125 GeV Higgs for supersymmetric models, Phys. Lett. B 708 (2012) 162 [arXiv:1112.3028] [INSPIRE].

[8] P. Draper, P. Meade, M. Reece and D. Shih, Implications of a $125 \mathrm{GeV}$ Higgs for the MSSM and low-scale SUSY breaking, Phys. Rev. D 85 (2012) 095007 [arXiv:1112.3068] [INSPIRE].

[9] M. Carena, S. Gori, N.R. Shah and C.E.M. Wagner, A $125 \mathrm{GeV}$ SM-like Higgs in the MSSM and the $\gamma \gamma$ rate, JHEP 03 (2012) 014 [arXiv:1112.3336] [INSPIRE].

[10] O. Buchmueller et al., Higgs and supersymmetry, Eur. Phys. J. C 72 (2012) 2020 [arXiv: 1112.3564] [INSPIRE].

[11] A. Arvanitaki and G. Villadoro, A non Standard Model Higgs at the LHC as a sign of naturalness, JHEP 02 (2012) 144 [arXiv:1112.4835] [INSPIRE].

[12] M. Papucci, J.T. Ruderman and A. Weiler, Natural SUSY endures, JHEP 09 (2012) 035 [arXiv:1110.6926] [INSPIRE].

[13] P.H. Chankowski, J.R. Ellis and S. Pokorski, The fine tuning price of LEP, Phys. Lett. B 423 (1998) 327 [hep-ph/9712234] [INSPIRE].

[14] R. Barbieri and A. Strumia, About the fine tuning price of LEP, Phys. Lett. B 433 (1998) 63 [hep-ph/9801353] [INSPIRE]. 
[15] G.L. Kane and S.F. King, Naturalness implications of LEP results, Phys. Lett. B 451 (1999) 113 [hep-ph/9810374] [INSPIRE].

[16] L. Giusti, A. Romanino and A. Strumia, Natural ranges of supersymmetric signals, Nucl. Phys. B 550 (1999) 3 [hep-ph/9811386] [INSPIRE].

[17] M. Maniatis, The next-to-minimal supersymmetric extension of the Standard Model reviewed, Int. J. Mod. Phys. A 25 (2010) 3505 [arXiv:0906.0777] [InSPIRE].

[18] U. Ellwanger, C. Hugonie and A.M. Teixeira, The next-to-minimal supersymmetric Standard Model, Phys. Rept. 496 (2010) 1 [arXiv:0910.1785] [InSPIRE].

[19] M. Bastero-Gil, C. Hugonie, S.F. King, D.P. Roy and S. Vempati, Does LEP prefer the NMSSM?, Phys. Lett. B 489 (2000) 359 [hep-ph/0006198] [INSPIRE].

[20] R. Dermisek and J.F. Gunion, Escaping the large fine tuning and little hierarchy problems in the next to minimal supersymmetric model and $h \rightarrow$ aa decays, Phys. Rev. Lett. 95 (2005) 041801 [hep-ph/0502105] [INSPIRE].

[21] R. Dermisek and J.F. Gunion, Consistency of LEP event excesses with an $h \rightarrow$ aa decay scenario and low-fine-tuning NMSSM models, Phys. Rev. D 73 (2006) 111701 [hep-ph/0510322] [INSPIRE].

[22] R. Dermisek and J.F. Gunion, The NMSSM solution to the fine-tuning problem, precision electroweak constraints and the largest LEP Higgs event excess, Phys. Rev. D 76 (2007) 095006 [arXiv: 0705 .4387] [INSPIRE].

[23] A. Delgado, C. Kolda, J.P. Olson and A. de la Puente, Solving the little hierarchy problem with a singlet and explicit $\mu$ terms, Phys. Rev. Lett. 105 (2010) 091802 [arXiv:1005.1282] [INSPIRE].

[24] U. Ellwanger, G. Espitalier-Noel and C. Hugonie, Naturalness and fine tuning in the NMSSM: implications of early LHC results, JHEP 09 (2011) 105 [arXiv:1107.2472] [INSPIRE].

[25] G.G. Ross and K. Schmidt-Hoberg, The fine-tuning of the generalised NMSSM, Nucl. Phys. B 862 (2012) 710 [arXiv:1108.1284] [INSPIRE].

[26] Z. Kang, J. Li and T. Li, On naturalness of the MSSM and NMSSM, JHEP 11 (2012) 024 [arXiv:1201.5305] [INSPIRE].

[27] J. Cao, Z. Heng, J.M. Yang and J. Zhu, Status of low energy SUSY models confronted with the LHC $125 \mathrm{GeV}$ Higgs data, JHEP 10 (2012) 079 [arXiv:1207.3698] [INSPIRE].

[28] M. Perelstein and B. Shakya, XENON100 implications for naturalness in the MSSM, NMSSM and $\lambda$-supersymmetry model, Phys. Rev. D 88 (2013) 075003 [arXiv:1208.0833] [INSPIRE].

[29] J.E. Kim and H.P. Nilles, The $\mu$ problem and the strong CP problem, Phys. Lett. B 138 (1984) 150 [INSPIRE].

[30] R. Barbieri, L.J. Hall, Y. Nomura and V.S. Rychkov, Supersymmetry without a light Higgs boson, Phys. Rev. D 75 (2007) 035007 [hep-ph/0607332] [INSPIRE].

[31] R. Barbieri, D. Buttazzo, K. Kannike, F. Sala and A. Tesi, Exploring the Higgs sector of a most natural NMSSM, Phys. Rev. D 87 (2013) 115018 [arXiv:1304.3670] [INSPIRE].

[32] V. Barger, P. Langacker, H.-S. Lee and G. Shaughnessy, Higgs sector in extensions of the MSSM, Phys. Rev. D 73 (2006) 115010 [hep-ph/0603247] [INSPIRE]. 
[33] U. Ellwanger and C. Hugonie, The upper bound on the lightest Higgs mass in the NMSSM revisited, Mod. Phys. Lett. A 22 (2007) 1581 [hep-ph/0612133] [INSPIRE].

[34] R. Dermisek and J.F. Gunion, A comparison of mixed-Higgs scenarios in the NMSSM and the MSSM, Phys. Rev. D 77 (2008) 015013 [arXiv:0709.2269] [INSPIRE].

[35] R. Barbieri, L.J. Hall, A.Y. Papaioannou, D. Pappadopulo and V.S. Rychkov, An alternative NMSSM phenomenology with manifest perturbative unification, JHEP 03 (2008) 005 [arXiv: 0712.2903] [INSPIRE].

[36] J.-J. Cao, Z.-X. Heng, J.M. Yang, Y.-M. Zhang and J.-Y. Zhu, A SM-like Higgs near $125 \mathrm{GeV}$ in low energy SUSY: a comparative study for MSSM and NMSSM, JHEP 03 (2012) 086 [arXiv:1202.5821] [INSPIRE].

[37] K.S. Jeong, Y. Shoji and M. Yamaguchi, Singlet-doublet Higgs mixing and its implications on the Higgs mass in the PQ-NMSSM, JHEP 09 (2012) 007 [arXiv: 1205.2486] [INSPIRE].

[38] K. Agashe, Y. Cui and R. Franceschini, Natural islands for a $125 \mathrm{GeV}$ Higgs in the scale-invariant NMSSM, JHEP 02 (2013) 031 [arXiv:1209.2115] [INSPIRE].

[39] K. Choi, S.H. Im, K.S. Jeong and M. Yamaguchi, Higgs mixing and diphoton rate enhancement in NMSSM models, JHEP 02 (2013) 090 [arXiv:1211.0875] [INSPIRE].

[40] K. Kowalska, S. Munir, L. Roszkowski, E.M. Sessolo, S. Trojanowski and Y.-L.S. Tsai, Constrained next-to-minimal supersymmetric Standard Model with a $126 \mathrm{GeV}$ Higgs boson: a global analysis, Phys. Rev. D 87 (2013) 115010 [arXiv:1211.1693] [INSPIRE].

[41] S.F. King, M. Mühlleitner, R. Nevzorov and K. Walz, Natural NMSSM Higgs bosons, Nucl. Phys. B 870 (2013) 323 [arXiv:1211. 5074] [INSPIRE].

[42] Z. Kang, J. Li, T. Li, D. Liu and J. Shu, Probing the CP-even Higgs sector via $\mathrm{H}_{3} \rightarrow \mathrm{H}_{2} \mathrm{H}_{1}$ in the natural next-to-minimal supersymmetric Standard Model, Phys. Rev. D 88 (2013) 015006 [arXiv: 1301.0453 ] [INSPIRE].

[43] N.D. Christensen, T. Han, Z. Liu and S. Su, Low-mass Higgs bosons in the NMSSM and their LHC implications, JHEP 08 (2013) 019 [arXiv: 1303.2113] [INSPIRE].

[44] T. Cheng, J. Li, T. Li and Q.-S. Yan, Natural NMSSM confronting with the LHC7-8, Phys. Rev. D 89 (2014) 015015 [arXiv: 1304.3182] [INSPIRE].

[45] M. Badziak, M. Olechowski and S. Pokorski, New regions in the NMSSM with a $125 \mathrm{GeV}$ Higgs, JHEP 06 (2013) 043 [arXiv: 1304.5437] [INSPIRE].

[46] C. Beskidt, W. de Boer and D.I. Kazakov, A comparison of the Higgs sectors of the CMSSM and NMSSM for a $126 \mathrm{GeV}$ Higgs boson, Phys. Lett. B 726 (2013) 758 [arXiv:1308.1333] [INSPIRE].

[47] K. Choi, S.H. Im, K.S. Jeong and M.-S. Seo, Higgs phenomenology in the Peccei-Quinn invariant NMSSM, JHEP 01 (2014) 072 [arXiv:1308.4447] [INSPIRE].

[48] J. Cao, F. Ding, C. Han, J.M. Yang and J. Zhu, A light Higgs scalar in the NMSSM confronted with the latest LHC Higgs data, JHEP 11 (2013) 018 [arXiv:1309.4939] [INSPIRE].

[49] G. Cacciapaglia, A. Deandrea, G.D. La Rochelle and J.-B. Flament, Searching for a lighter Higgs boson: parametrization and sample tests, Phys. Rev. D 91 (2015) 015012 [arXiv: 1311.5132] [INSPIRE]. 
[50] C. Englert et al., Precision measurements of Higgs couplings: implications for new physics scales, J. Phys. G 41 (2014) 113001 [arXiv:1403.7191] [INSPIRE].

[51] U. Ellwanger and C. Hugonie, The semi-constrained NMSSM satisfying bounds from the LHC, LUX and Planck, JHEP 08 (2014) 046 [arXiv: 1405.6647] [INSPIRE].

[52] M. Badziak, M. Olechowski and S. Pokorski, Experimental signatures of a light singlet-like scalar in NMSSM, arXiv:1406.1492 [INSPIRE].

[53] K.S. Jeong, Y. Shoji and M. Yamaguchi, Higgs mixing in the NMSSM and light Higgsinos, JHEP 11 (2014) 148 [arXiv: 1407.0955] [INSPIRE].

[54] S.F. King, M. Mühlleitner, R. Nevzorov and K. Walz, Discovery prospects for NMSSM Higgs bosons at the high-energy Large Hadron Collider, Phys. Rev. D 90 (2014) 095014 [arXiv: 1408.1120] [INSPIRE].

[55] B. Allanach, M. Badziak, C. Hugonie and R. Ziegler, Light sparticles from a light singlet in gauge mediation, Phys. Rev. D 92 (2015) 015006 [arXiv: 1502.05836] [INSPIRE].

[56] D. Buttazzo, F. Sala and A. Tesi, Singlet-like Higgs bosons at present and future colliders, JHEP 11 (2015) 158 [arXiv:1505.05488] [INSPIRE].

[57] M. Guchait and J. Kumar, Light Higgs bosons in NMSSM at the LHC, arXiv:1509.02452 [INSPIRE].

[58] F. Domingo and G. Weiglein, NMSSM interpretations of the observed Higgs signal, arXiv: 1509.07283 [INSPIRE].

[59] Delphi, OPAL, ALEPH, LEP Working Group for Higgs Boson Searches and L3 collaborations, S. Schael et al., Search for neutral MSSM Higgs bosons at LEP, Eur. Phys. J. C 47 (2006) 547 [hep-ex/0602042] [INSPIRE].

[60] ATLAS collaboration, Projections for measurements of Higgs boson cross sections, branching ratios and coupling parameters with the ATLAS detector at a HL-LHC, ATL-PHYS-PUB-2013-014, CERN, Geneva Switzerland (2013).

[61] CMS collaboration, Projected performance of an upgraded CMS detector at the LHC and HL-LHC: contribution to the Snowmass process, arXiv:1307.7135 [INSPIRE].

[62] ATLAS collaboration, Search for scalar diphoton resonances in the mass range 65-600 GeV with the ATLAS detector in pp collision data at $\sqrt{s}=8 \mathrm{TeV}$, Phys. Rev. Lett. 113 (2014) 171801 [arXiv: 1407.6583 ] [INSPIRE].

[63] CMS collaboration, Search for new resonances in the diphoton final state in the mass range between 80 and $110 \mathrm{GeV}$ in pp collisions at $\sqrt{s}=8 \mathrm{TeV}$, CMS-HIG-14-037, CERN, Geneva Switzerland (2014).

[64] S. Moretti and S. Munir, Di-photon Higgs signals at the LHC in the next-to-minimal supersymmetric Standard Model, Eur. Phys. J. C 47 (2006) 791 [hep-ph/0603085] [INSPIRE].

[65] U. Ellwanger, Enhanced di-photon Higgs signal in the next-to-minimal supersymmetric Standard Model, Phys. Lett. B 698 (2011) 293 [arXiv:1012.1201] [INSPIRE].

[66] J. Cao, Z. Heng, T. Liu and J.M. Yang, Di-photon Higgs signal at the LHC: a comparative study in different supersymmetric models, Phys. Lett. B 703 (2011) 462 [arXiv:1103.0631] [INSPIRE]. 
[67] R. Benbrik, M. Gomez Bock, S. Heinemeyer, O. Stål, G. Weiglein and L. Zeune, Confronting the MSSM and the NMSSM with the discovery of a signal in the two photon channel at the LHC, Eur. Phys. J. C 72 (2012) 2171 [arXiv:1207.1096] [InSPIRE].

[68] Z. Heng, A $125 \mathrm{GeV}$ Higgs and its di-photon signal in different SUSY models: a mini review, Adv. High Energy Phys. 2012 (2012) 312719 [arXiv:1210.3751] [INSPIRE].

[69] J.-W. Fan et al., Study of diphoton decays of the lightest scalar Higgs boson in the next-to-minimal supersymmetric Standard Model, Chin. Phys. C 38 (2014) 073101 [arXiv: 1309.6394] [INSPIRE].

[70] J. Bernon, B. Dumont and S. Kraml, Status of Higgs couplings after Run 1 of the LHC, Phys. Rev. D 90 (2014) 071301 [arXiv:1409.1588] [inSPIRE].

[71] O. Stål, G. Weiglein and L. Zeune, Improved prediction for the mass of the $W$ boson in the NMSSM, JHEP 09 (2015) 158 [arXiv:1506.07465] [INSPIRE].

[72] T. Robens and T. Stefaniak, Status of the Higgs singlet extension of the Standard Model after LHC Run 1, Eur. Phys. J. C 75 (2015) 104 [arXiv:1501.02234] [InSPIRE].

[73] A. Falkowski, C. Gross and O. Lebedev, A second Higgs from the Higgs portal, JHEP 05 (2015) 057 [arXiv: 1502.01361] [INSPIRE].

[74] M. Gorbahn, J.M. No and V. Sanz, Benchmarks for Higgs effective theory: extended Higgs sectors, JHEP 10 (2015) 036 [arXiv: 1502.07352] [INSPIRE].

[75] S.I. Godunov, A.N. Rozanov, M.I. Vysotsky and E.V. Zhemchugov, Extending the Higgs sector: an extra singlet, Eur. Phys. J. C 76 (2016) 1 [arXiv: 1503.01618] [INSPIRE].

[76] U. Ellwanger, J.F. Gunion and C. Hugonie, NMHDECAY: a fortran code for the Higgs masses, couplings and decay widths in the NMSSM, JHEP 02 (2005) 066 [hep-ph/0406215] [INSPIRE].

[77] U. Ellwanger and C. Hugonie, NMHDECAY 2.0: an updated program for sparticle masses, Higgs masses, couplings and decay widths in the NMSSM, Comput. Phys. Commun. 175 (2006) 290 [hep-ph/0508022] [INSPIRE].

[78] G. Degrassi and P. Slavich, On the radiative corrections to the neutral Higgs boson masses in the NMSSM, Nucl. Phys. B 825 (2010) 119 [arXiv:0907.4682] [INSPIRE].

[79] R.V. Harlander, S. Liebler and H. Mantler, SusHi: a program for the calculation of Higgs production in gluon fusion and bottom-quark annihilation in the Standard Model and the MSSM, Comput. Phys. Commun. 184 (2013) 1605 [arXiv:1212.3249] [INSPIRE].

[80] M. Carena, H.E. Haber, I. Low, N.R. Shah and C.E.M. Wagner, On the alignment limit of the NMSSM Higgs sector, arXiv: 1510.09137 [INSPIRE].

[81] A. Djouadi and J. Quevillon, The MSSM Higgs sector at a high $M_{\mathrm{SUSY}}$ : reopening the low $\tan \beta$ regime and heavy Higgs searches, JHEP 10 (2013) 028 [arXiv:1304.1787] [INSPIRE].

[82] A. Djouadi, L. Maiani, A. Polosa, J. Quevillon and V. Riquer, Fully covering the MSSM Higgs sector at the LHC, JHEP 06 (2015) 168 [arXiv: 1502.05653] [INSPIRE].

[83] N.-E. Bomark, S. Moretti, S. Munir and L. Roszkowski, A light NMSSM pseudoscalar Higgs boson at the LHC redux, JHEP 02 (2015) 044 [arXiv: 1409.8393] [INSPIRE]. 